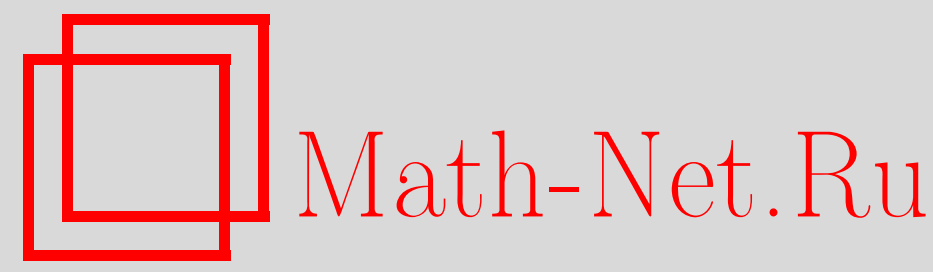

В. З. Гринес, О топологической классификации структурно устойчивых диффеоморфизмов поверхностей с одномерными аттракторами и репеллерами, Матем. сб., 1997, том 188, номер 4, 57-94

DOI: https://doi.org/10.4213/sm216

Использование Общероссийского математического портала Math-Net.Ru подразумевает, что вы прочитали и согласны с пользовательским соглашением http://www . mathnet.ru/rus/agreement

Параметры загрузки:

IP: 54.89 .56 .158

26 апреля 2023 г., 15:49:19 
УДК 513.83

\author{
В.3. Гринес
}

\title{
О топологической классификации структурно устойчивых диффеоморфизмов поверхностей с одномерными аттракторами и репеллерами
}

\footnotetext{
В настоящей работеустанавливаются необходимые и достаточные условия топологической сопряженности структурно устойчивых сохраняющих ориентацию диффеоморфизмов из класса $S(M)$, заданных на двумерном гладком замкнутом ориентируемом многообразии $M$ и удовлетворяющих следующим условиям:

1) каждое нетривиальное базисное множество диффеоморфизма $f \in S(M)$ есть одномерный аттрактор или репеллер;

2) множество гетероклинических траекторий, принадлежащих пересечению устойчивых и неустойчивых многообразий седловых периодических точек из тривиальных базисных множеств, конечно.

Библиограф̆фия: 35 названий.
}

\section{§1. Введение. Предварительные сведения и формулировка результатов}

Возможность топологической классификации диффеоморфизмов из класса $S(M)$ основана на полученной к настоящему времени топологической классификации диффеоморфизмов Морса-Смейла с конечным множеством гетероклинических траекторий и топологической классификации одномерных базисных множеств $A$-диффеоморфизмов, т.е. диффеоморфизмов, удовлетворяющих аксиоме $(A)$ С. Смейла.

Как известно, необходимым и достаточньм условием структурной устойчивости диффеоморфизма, заданного на двумерном многообразии, является выполнение для него аксиомы $(A)$ С. Смейла и строгого условия трансверсальности (см. [1]-[6]). Согласно теореме С. Смейла о спектральном разложении множество неблуждающих точек $A$-диффеоморфизма $f$ представляется в виде конечного объединения попарно непересекающихся замкнутых инвариантных множеств, называемых базисными множествами, каждое из которых содержит всюду плотную траекторию [20]. Базисное множество, являющееся периодической траекторией, будем называть тривиальным, а базисное множество, не совпадающее с периодической траекторией, будем называть нетривиальным. Если неблуждающие множества $A$-диффеоморфизмов содержат нетривиальные одномерные базисные

Работа вьполнена при частичной финансовой поддержке Российского фонда фундаментальных исследований (грант № 96-01-00236). 
множества, то при решении задачи топологической классификации таких диффеоморфизмов естественно вначале появляется задача о топологической сопряженности ограничений $A$-диффеоморфизмов на одномерные базисные множества, которая формулируется следующим образом.

Пусть $\Omega, \Omega^{\prime}$ - одномерные базисные множества А-диффеоморфизмов $f, f^{\prime}$, соответственно. Требуется найти необходимые и достаточные условия существования гомеоморфизма $g: M \rightarrow M$ такого, что $g(\Omega)=\Omega^{\prime}$ и

$$
\left.f^{\prime}\right|_{\Omega}=\left.g f g^{-1}\right|_{\Omega^{\prime}}
$$

Первые результаты в решении этой задачи были получены автором настояшей работы для ориентируемых аттракторов (см. [8]-[10]) ${ }^{1}$ и затем были обобщены P.В. Плыкиным на случай просторно расположенных аттракторов и аттракторов со связками степени не больше двух $[11]^{2}$. Поставленная задача была полностью решена автором и Х. Х. Калаем для произвольных аттракторов на ориентируемой поверхности $M$ рода $p \geqslant 0$ [12], [13] (см. также обзоры [14], [15, гл. 3]). В недавнее время появился цикл работ А. Жирова [16]-[18], посвященный комбинаторному описанию аттракторов.

В [19] (см. также [15]) автором настоящей работы найдены необходимые и достаточные условия топологической сопряженности сохраняющих ориентацию диффеоморфизмов Морса-Смейла, заданных на двумерном гладком замкнутом ориентируемом многообразии в предположении, что множество гетероклинических траекторий рассматриваемых диффеоморфизмов конечно ${ }^{3}$.

Изученньй в [19] класс диффеоморфизмов содержит класс градиентноподобных диффеоморфизмов и класс диффеоморфизмов с ориентируемьпи гетероклиническими множествами, для которых найден полный топологический инвариант в работах [21]-[24]. В работах [25]-[28] рассмотрены некоторые частные случаи диффеоморфизмов Морса-Смейла с конечньм и счетным множеством гетероклинических траекторий на $M$.

\footnotetext{
${ }^{1}$ Нетривиальное базисное множество $\Omega$ называется ориентируемым, если для любой точки $x \in \Omega$ и любых фиксированных положительных чисел $\alpha, \beta$ индекс пересечения многообразий $W_{\alpha}^{s}(x), W_{\beta}^{u}(x)$ один и тот же во всех точках пересечения, где $W_{\alpha}^{s}(x)=$ $\left\{y \in W^{s}(x) \mid l(x, y)<\alpha\right\}, W_{\beta}^{u}(x)=\left\{y \in W^{u}(x) \mid l(x, y)<\beta\right\} \quad(l$ - метрика на $\left.W^{s}(x), W^{u}(x)\right)$.

${ }^{2}$ Нетривиальное базисное множество $\Omega$ называется просторно расположсенным, если не существует гомотопной нулю петли, образованной парой отрезков устойчивого и неустойчивого многообразия какой-либо точки из $\Omega$.

${ }^{3}$ Согласно [20] класс $G(M)$ дифффеоморфизмов Морса-Смейла выделяется следующими условиями:

1) множество неблуждающих траекторий диффеоморфизма $f \in G(M)$ конечно;

2) все периодические точки диффеоморфизма $f \in G(M)$ гиперболичны;

3) для любой пары периодических точек $p, q$ диффеоморфизма $f \in G(M)$ пересечение $W^{s}(p)$ и $W^{u}(q)$ либо пусто, либо трансверсально.
}

В силу [1] класс диффеоморфизмов Морса-Смейла состоит из структурно устойчивых диффеоморфизмов. 
Приведем некоторые факты о топологической структуре нетривиальных базисных множеств размерности нуль или единица $A$-диффеоморфизма $f$, заданного на двумерном многообразии $M .{ }^{4}$

Согласно Д.В. Аносову [30] и Р. Боуэну [31] нетривиальное базисноемножество $\Omega$ представляется в виде конечного объединения $\Omega_{1} \cup \cdots \cup \Omega_{m}$ связных замкнутых подмножеств $(m \geqslant 1)$, называемых $C$-плотныцми компонентами множества $\Omega$, таких, что $f^{m}\left(\Omega_{i}\right)=\Omega_{i}, f\left(\Omega_{i}\right)=\Omega_{i+1}, \Omega_{m+1}=\Omega_{1}$. Для каждой точки $x$, принадлежашей $C$-плотной компоненте $\Omega_{i}, i \in\{1, \ldots, m\}$, множество $W^{\sigma}(x) \cap \Omega_{i}$ плотно в $\Omega_{i}, \sigma \in\{s, u\}$. В силу [9] каждая компонента множества $W^{\sigma}(x) \backslash x$, не содержащая граничной периодической точки, также содержит множество плотное в $\Omega_{i}$.

Для $\sigma \in\{s, u\}$ положим $\bar{\sigma}=s$, если $\sigma=u$, и $\bar{\sigma}=u$, если $\sigma=s$. Периодическая точка $p$, принадлежащая нетривиальному базисному множеству $\Omega$, называется граничной периодической точкой типа $\sigma$, если одна из компонент связности множества $W^{\sigma}(p) \backslash p$ не пересекается с $\Omega$, а обе компоненты связности множества $W^{\bar{\sigma}}(p) \backslash p$ пересекаются с $\Omega$. Периодическая точка $p \in \Omega$ называется граничной периодической точкой типа $(s, u)$, если одна из компонент связности каждого из множеств $W^{s}(p) \backslash p, W^{u}(p) \backslash p$ не пересекается с $\Omega$. Если множество $\Omega$ нульмерно или одномерно, то множество граничных периодических точек не пусто и конечно [8]-[11], [33] (см. также [14], [15, гл. 3]).

Если базисное множество одномерно, то в силу [32] оно является аттрактором или репеллером, и для любой точки $x$ аттрактора (репеллера) $\Omega$ неустойчивое (устойчивое) многообразие принадлежит $\Omega$.

Если $p$ граничная периодическая точка аттрактора (репеллера) $\Omega$, то будем обозначать через $W_{\varnothing}^{s}(p)\left(W_{\varnothing}^{u}(p)\right)$ компоненту связности множества $W^{s}(p) \backslash p$ $\left(W^{u}(p) \backslash p\right)$, не пересекающуюся с $\Omega$, и через $W_{\infty}^{s}(p)\left(W_{\infty}^{u}(p)\right)$ - компоненту связности множества $W^{s}(p) \backslash p\left(W^{u}(p) \backslash p\right)$, пересекающуюся с $\Omega$. Периодическую точку одномерного аттрактора, не являюшуюся граничной, будем называть внутренней.

Если $q$ седловая периодическая точка диффеоморфизма $f$ и $x, y \in W^{\sigma}(q)$, $\sigma \in\{s, u\}$, то будем обозначать через $[x, y]^{\sigma},[x, y)^{\sigma},(x, y]^{\sigma},(x, y)^{\sigma}$ связные дуги на многообразии $W^{\sigma}(q)$ с граничными точками $x, y$.

Из работ [8]-[11], [33] (см. также обзоры [14], [15, гл. 3]) следует, что для одномерного аттрактора (репеллера) $\Omega$ достижимая изнутри граница множества $M \backslash \Omega_{i}$ распадается единственным образом на конечное число $R(\Omega)$ связок (понятие связки принадлежит Р.В. Плыкину [11]). Каждая связка $C$ аттрактора (репеллера) есть объединение $r_{C}$ неустойчивых (устойчивых) многообразий $W^{\sigma}\left(p_{1}\right) \cup \cdots \cup W^{\sigma}\left(p_{r_{C}}\right)$ граничных периодических точек $p_{1}, \ldots, p_{r_{C}}$ множества $\Omega, \sigma=u(\sigma=s)$, обладающих следуюшим свойством: сушествует последовательность точек $x_{1}, \ldots, x_{2 r_{C}}$ такая, что

\footnotetext{
${ }^{4}$ Если размерность базисного множества равна двум, то оно совпадает со всем многообразием $M$, котороев этом случае является тором, а сам диффеоморфизм $f-\mathrm{У}$-диффеоморфизмом Д. В. Аносова [29].
} 
1) $x_{2 i-1}, x_{2 i}$ принадлежат различным компонентам связности множества $W^{\sigma}\left(p_{i}\right) \backslash p_{i} ;$

2) $x_{2 i+1} \in W^{\bar{\sigma}}\left(x_{2 i}\right)$ (полагаем $\left.x_{2 r_{C}+1}=x_{1}\right)$;

3) $\left(x_{2 i}, x_{2 i+1}\right)^{\bar{\sigma}} \cap \Omega=\varnothing, i=\overline{1, r_{C}}$;

4) кривая $L_{2 i}^{\sigma} \cup\left(x_{2 i}, x_{2 i+1}\right)^{\bar{\sigma}} \cup L_{2 i+1}^{\sigma}$ является достижимой изнутри гранищей области, гомеоморфной открытому диску $D_{i}$, где $L_{2 i}^{\sigma}\left(L_{2 i+1}^{\sigma}\right)$ есть компонента связности множества $W^{\sigma}\left(x_{2 i}\right) \backslash x_{2 i}\left(W^{\sigma}\left(x_{2 i+1}\right) \backslash x_{2 i+1}\right)$, не содержащая точки $p_{i}\left(p_{i+1}\right)$.

На рис. 1а-1в изображены связки степени 1-3.

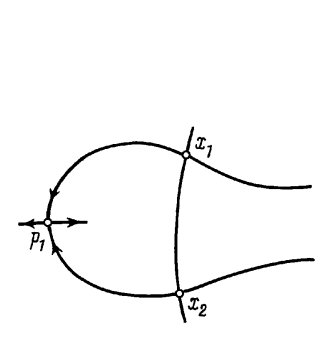

$a$
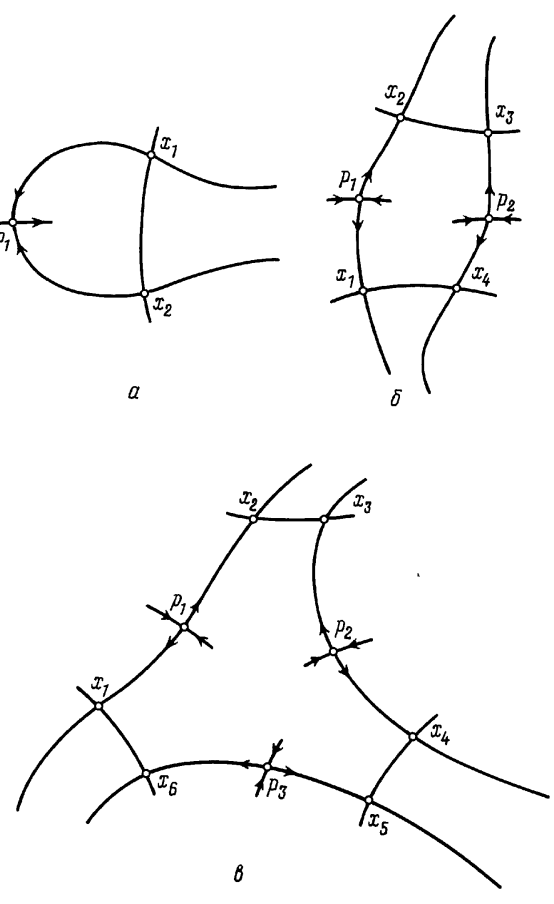

Рис. 1

Пусть $p$ граничная периодическая точка аттрактора (репеллера) $\Omega$ и $x \in W^{\sigma}(p)$ любая точка отличная от $p$, где $\sigma=u(\sigma=s)$. Обозначим через $W_{x}^{\sigma}(p)$ компоненту связности множества $W^{\sigma}(p) \backslash p$, содержащую точку $x$. Из сказанного выше следует, что сушествует единственная граничная периодическая точка $q$ ( $q$ может совпадать с $p$, если $p$ принадлежит связке степени 1) такая, что для точки $x \in W_{x}^{\sigma}(p)$ найдется единственная точка $y$, отличная от $x, y \in W^{\bar{\sigma}}(x) \cap W^{\sigma}(q)$ и $(x, y)^{\bar{\sigma}} \cap \Omega=\varnothing$ (если $q$ совпадает с $p$, то $y$ принадлежит компоненте связности множества $W^{\sigma}(p) \backslash p$, не содержашей точки $\left.x\right)$. При этом для любой точки $\widetilde{x} \in W_{x}^{\sigma}(p)$ найдется единственная точка $\widetilde{y} \in W_{y}^{\sigma}(q), \widetilde{y} \in W^{\bar{\sigma}}(\widetilde{x}) \cap W^{\sigma}(q)$ и $(\widetilde{x}, \widetilde{y})^{\bar{\sigma}} \cap \Omega=\varnothing$. Положим $(\widetilde{x}, \widetilde{y})^{\bar{\sigma}}=\Delta^{\bar{\sigma}}(\widetilde{x})$ и назовем компоненты $W_{x}^{\sigma}(p), W_{y}^{\sigma}(q) \bar{\sigma}$-связанными (рис. 2а и 2б). 


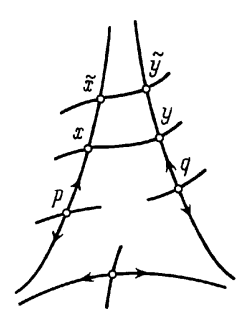

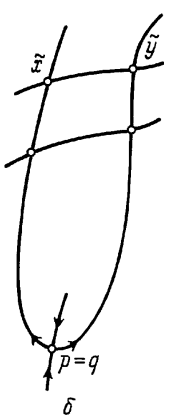

Рис. 2

Обозначим через $L^{\sigma}(x)\left(L^{\sigma}(y)\right)$ компоненту связности множества $W^{\sigma}(x) \backslash x$ $\left(W^{\sigma}(y) \backslash y\right)$, которая не содержит точки $p(q)$. Положим $l_{x y}=L^{\sigma}(x) \cup L^{\sigma}(y) \cup$ $[x, y]^{\bar{\sigma}}$. Тогда множество $D_{x y}=\bigcup_{\widetilde{x} \in L^{\sigma}(x)} \Delta^{\bar{\sigma}}(\widetilde{x})$ есть область, которая обладает следующими свойствами:

a) достижимая изнутри граница области $D_{x y}$ есть $l_{x y}$;

б) $D_{x y}$ гомеоморфна открытому диску;

в) $D_{x y} \cup(x, y)^{\bar{\sigma}}$ состоит из блуждаюших точек диффеоморфизма $f$ (рис. $2 \mathrm{a}$ и $2 б)$.

Положим $D_{p q x y}=\bigcup_{\widetilde{x} \in W_{x}^{\sigma}(p)} \Delta^{\bar{\sigma}}(\widetilde{x})$.

Для граничной периодической точки $p$, принадлежащей аттрактору (репеллеру) $\Omega$, положим $\partial W_{\varnothing}^{\sigma}(p)=\overline{W_{\varnothing}^{\sigma}(p)} \backslash\left(W_{\varnothing}^{\sigma}(p) \cup p\right)$, где $\sigma=s(\sigma=u)$.

Для седловой периодической точки $q$, принадлежашей тривиальному базисному множеству диффеоморфизма $f$, положим $\partial W^{\sigma}(q)=\overline{W^{\sigma}(q)} \backslash\left(W^{\sigma}(q) \cup q\right)$, где $\sigma \in\{s, u\}$.

ЛЕмма 1. Пусть $f$ структурно устойчивый диффеоморфизм, заданный на гладком замкнутом двумерном ориентируемом многообразии $M$ рода $g \geqslant 0$, неблуждающее множество которого содержит одномерное базисное множество $\Omega$, компоненты $W_{x}^{\sigma}(p), W_{y}^{\sigma}(q) \bar{\sigma}$-связаны, где $\sigma=u$, если $\Omega$ есть аттрактор, $и \sigma=s$, если $\Omega$ есть репеллер $\left(x \in W^{\sigma}(p), y \in W^{\sigma}(q), p, q\right.$ граничнве периодические точки множества $\Omega$, причем если р совпадает с $q$, то $x, y$ принадлежат различным компонентам связности множества $\left.W^{\sigma}(p) \backslash p\right)$. Тогда существуют $l \geqslant 1$ источниковых, если $\sigma=u$, стоковых, если $\sigma=s$, периодических точек $\alpha_{1}^{\sigma}, \ldots, \alpha_{l}^{\sigma}$ и $n \geqslant 0$ седловых периодических точек $p_{1}, \ldots, p_{n}$ (если $n=0$, то $l=1)$, принадлежащих тривиальным базисным множсествам, таких, что

1) множество

$$
L_{p q}= \begin{cases}W_{\varnothing}^{\bar{\sigma}}(p) \cup W_{\varnothing}^{\bar{\sigma}}(q) \cup \alpha_{1}^{\sigma} \cup p \cup q, & \text { если } n=0, \\ W_{\varnothing}^{\bar{\sigma}}(p) \cup W_{\varnothing}^{\bar{\sigma}}(q) \cup \bigcup_{i=1}^{n} W^{\bar{\sigma}}\left(p_{i}\right) \cup \bigcup_{i=1}^{l} \alpha_{i}^{\sigma} \cup p \cup q, & \text { еслu } n \geqslant 1,\end{cases}
$$

является связным одномерным комплексом; 
2) каждое из множеств $\partial W_{\varnothing}^{\bar{\sigma}}(p), \partial W_{\varnothing}^{\bar{\sigma}}(q)$ состоит в точности из одной источниковой, если $\sigma=u$, стоковой, если $\sigma=s$, периодической точки, принадлежсащей $L_{p q}$;

3) кажсде из мнохсеств $\partial W^{\bar{\sigma}}\left(p_{i}\right), i \in\{1, n\}$, состоит в точности из одной или двух источниковьх, если $\sigma=u$, стоковых, если $\sigma=s$, периодических точек, принадлежсащих $L_{p q}$;

4) комплекс $L_{p q}$ является топологическим пределом последовательнос$m u$ дyг $\left.f^{\nu N k}([x, y])^{\bar{\sigma}}\right)$ nрu $k \rightarrow+\infty$, где $\nu=-1$, если $\sigma=u, u$ $\nu=+1$, если $\sigma=$ s $и N \in \mathbb{Z}^{+}$mакое, что $f^{N}\left(W_{x}^{\sigma}(p)\right)=W_{x}^{\sigma}(p)$, $f^{N}\left(W_{x}^{\sigma}(q)\right)=W_{x}^{\sigma}(q)$

5) обгединение $L_{p q} \cup W_{x}^{\sigma}(p) \cup W_{y}^{\sigma}(q)$ является достижимой изнутри гра-

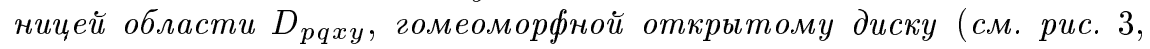
на котором рассмотрен случай, когда базисное множсество $\Omega$ есть аттрактор $(\sigma=u) ; n=0-$ puc. $3 \mathrm{a}, n>0-p u c .3 б)$.
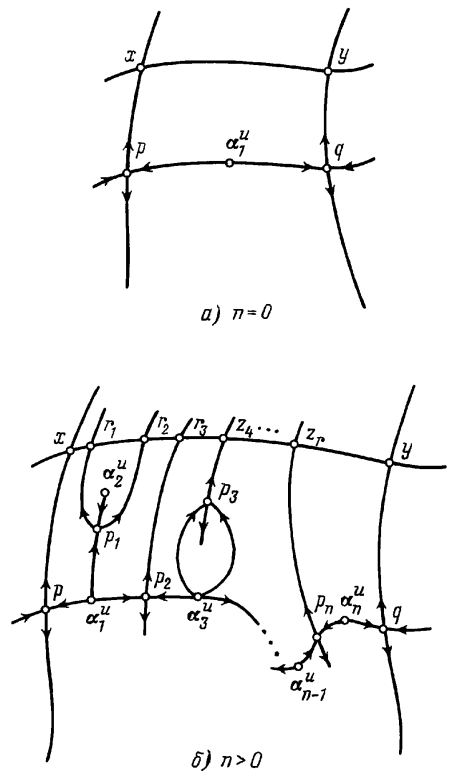

Рис. 3

Из леммы 1 непосредственно следует

ТЕОРЕма 1. Если неблуждающее множество структурно устойчивого диффеоморфизма $f$ содержит одномерный аттрактор (репеллер), тогда оно содерэсит и источниковую (стоковую) периодическую точку.

В [33] доказано, что утверждение теоремы 1 справедливо для сферы и тора и в случае, когда диффеоморфизм $f$ не является структурно устойчивьм, а лишь удовлетворяет аксиоме $(A) \mathrm{C}$. Смейла. Если же многообразие $M$ имеет род, больший 
чем единица, то условие структурной устойчивости $A$-диффеоморфизма $f$ существенно для вьполнения утверждения теоремы 1. В работе [34] приведен пример структурно неустойчивого $A$-диффеоморфизма двумерного многообразия рода 2 (кренделя), неблуждающее множество которого состоит в точности из одного одномерного аттрактора и одного одномерного репеллера.

Для базисного множества $\Omega$ обозначим через $\Gamma_{i}$ объединение всех его граничных периодических точек, принадлежащих $i$-й $C$-плотной компоненте.

Лемма 2. Пусть $\Omega$ аттрактор (репеллер) структурно устойчивого диффеоморфизма $f$, состоящий из $m C$-плотных компонент $\Omega_{1}, \ldots, \Omega_{m}$. Тогда существует инвариантное открытое множество $M_{\Omega}$, являющееся обтединением $m$ открытых подмножеств $M_{1}, \ldots, M_{m}$, такое, что

1) $\Omega_{i} \subset M_{i} \cup \Gamma_{i}, f^{m}\left(M_{i}\right)=M_{i}$;

2) $M_{\Omega} \backslash \Omega$ не содержит точек неблуждающего множества диффеоморфизма $f$;

3) граница множества $M_{\Omega}$ является одномерным комплексом, состоящим из множества граничных периодических точек множества $\Omega$ и компонент связности их устойчивых (неустойчивых) многообразий, непересекающихся $c \Omega$, устойчивых (неустойчивых) многообразий седловых периодических точек из тривиальных базисных множеств и конечного числа неустойчивых (устойчивых) периодических точек.

ОПРЕДЕЛЕНИЕ 1. Множество $M_{\Omega}$ будем называть канонической окрестностью базисного мноэсества $\Omega$.

Обозначим через $\Lambda$ множество, состоящее из объединения всех нетривиальных (одномерных) базисных множеств диффеоморфизма $f$, и через $M_{\Lambda}$ множество, состоящее из объединения канонических окрестностей всех базисных множеств из $\Lambda$.

Обозначим через $P$ множество периодических точек диффеоморфизма $f$, принадлежаших тривиальным базисным множествам, и через $P_{1}$ подмножество множества $P$, состоящее из всех седловых периодических точек.

Лемма 3. Пусть $f$ структурно устойчивъи ииффеоморфизм, $f \in S(M), u$ $L^{\sigma}(p)$ одна из компонент связности множества $W^{\sigma}(p) \backslash p, p \in P_{1}$. Тогда

1) ecлu $L^{\sigma}(p) \cap M_{\Lambda} \neq \varnothing$, mo $\partial L^{\sigma}(p)\left(\partial L^{\sigma}(p)=\overline{L^{\sigma}(p)} \backslash\left(L^{\sigma}(p) \cup p\right)\right)$ cocmoum в точности из одной $C$-плотной компоненты, либо некоторого одномерного аттрактора (если $\sigma=u$ ), либо одномерного репеллера (если $\sigma=s)$, и точка р принадлежит граниче множества $M_{\Lambda}$;

2) если $L^{\sigma}(p) \cap M_{\Lambda}=\varnothing и L^{\sigma}(p)$ не содержит гетероклинических точек, то $\partial L^{\sigma}(p)$ состоит либо из единственной стоковой (если $\left.\sigma=u\right)$, либо из единственной источниковой (если $\sigma=s)$ периодической точки;

3) если $L^{\sigma}(p) \cap M_{\Lambda}=\varnothing и L^{\sigma}(p)$ содержит гетероклинические точки, то $\partial L^{\sigma}(p)$ является связным одномерным комплексом $\overline{\bigcup_{q} W^{\sigma}(q)}$, дде обгединение берется по всем точкам $q \in P_{1}$, для которых $W^{\bar{\sigma}}(q) \cap$ $L^{\sigma}(p) \neq \varnothing$, при этом $W^{\sigma}(q)$ не содержит гетероклинических точек. 
ЛЕмма 4. Пусть $\Omega$ аттрактор (репеллер) структурно устойчивого диффеоморфизма $f$, состоящий из $m C$-плотных компонент $\Omega_{1}, \ldots, \Omega_{m}$, каждая из которых содержит набор $\widehat{C}_{i}$, состоящий из $R(\Omega)$ связок степени $r_{C}$ для $C \in \widehat{C}_{i}$. Тогда существуют окрестность $V$ множсества $\Omega$, являющаяся обвединением $m$ окрестностей $V_{i}$ компонент $\Omega_{i}$, компактное подмногообразие $N_{\Omega} \subset \bar{M}_{\Omega}$, являющееся облединением $m$ компактных двумерных подмногообразий с краем $N_{1}, \ldots, N_{m}$, и диффеоморфизм $f_{\Omega}$ подмногообразия $N_{\Omega}$ на себя такие, что

1) $\Omega_{i} \subset V_{i} \subset N_{i} \subset \bar{M}_{i}$;

2) $\left.f_{\Omega}\right|_{V}=\left.f\right|_{V}$

3) каждде подмногообразие $N_{i}$ имеет $R(\Omega)$ компонент края, род $q \geqslant 0$ u отрицательную эйлерову характеристику $\chi\left(N_{i}\right)=2-2 q-R(\Omega)$ (числа $q$ и $R(\Omega)$ однозначно определяются по $\Omega)$;

4) множсество $N_{\Omega} \backslash\left(\Omega \cup \partial N_{\Omega}\right)$ состоит из блужсдающих точек диффеоморфизма $f_{\Omega}$ и является обвединением $m R(\Omega)$ непересекающихся межсду собой областей, гомеоморфных кольиу; достижимая изнутри граница каждой такой области состоит в точности из одной связки $C$ множества $\Omega$ и одной компоненты края $\partial N_{\Omega}$ многообразия $N_{\Omega}$, содержащей в точности $r_{C}$ седловьх и $r_{C}$ источниковых (стоковьх) периодических точек диффеоморфизма $f_{\Omega}$ (рис. 4).

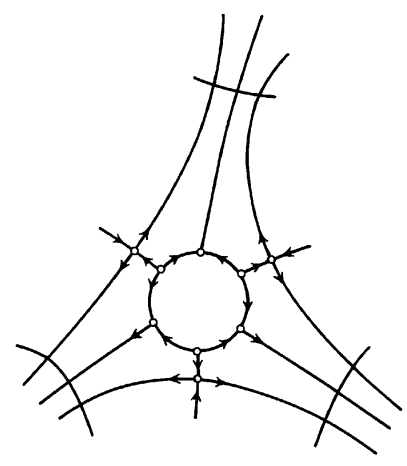

Рис. 4

ОПРЕДЕЛЕНИЕ 2. Подмногообразие $N_{\Omega}$ будем называть каноническим носителем, а пару $\left(N_{\Omega}, f_{\Omega}\right)$ - канонической формой аттрактора (репеллера) $\Omega$.

Зафиксируем номер $i \in\{1, \ldots, m\}$. Так как эйлерова характеристика подмногообразия $N_{i}$ отрицательна, то на плоскости Лобачевского, в реализации Пуанкаре на внутренности круга $|z|<1$ комплексной $z$-плоскости, существуют дискретная группа движений $F$, изоморфная фундаментальной группе подмногообразия $N_{i}$, и подмножество $H_{F}$ такие, что фактормножество $H_{F} / F$ гомеоморфно подмногообразию $N_{i}$. Обозначим через $\pi_{i}$ естественную проекцию $H_{F} \Rightarrow N_{i}$ и через $f_{i}$ ограничение диффеоморфизма $f_{\Omega}^{m}$ на подмногообразие $N_{i}$. Пусть $\bar{f}_{i}$ отображение $H_{F}$ на себя, накрываюшее диффеоморфизм $f_{i}$, т.е. $\bar{f}_{i}$ удовлетворяет условию 
$\pi_{i} \bar{f}_{i}=f_{i} \pi_{i}$. Отображение $\bar{f}_{i}$ индуцирует автоморфизм $\bar{f}_{i *}$ группы $F$ на себя по формуле $\bar{f}_{i *}(\gamma)=\bar{f}_{i} \gamma \bar{f}_{i}^{-1}, \gamma \in F$.

Если $\widetilde{\bar{f}}_{i}$ отображение $H_{F}$ на себя, накрьвающее диффеоморфизм $f_{i}$ и отличное от $\bar{f}_{i}$, тогда существует элемент $\alpha \in F$ такой, что $\widetilde{\bar{f}}_{i}=\alpha \bar{f}_{i}$. Отображение $\widetilde{\bar{f}}_{i}$ индуцирует автоморфизм $A_{\alpha} \bar{f}_{i *}$, где $A_{\alpha}$ - внутренний автоморфизм групшы $F$, задаваемый формулой $A_{\alpha}(\beta)=\alpha \beta \alpha^{-1}$.

Таким образом, каждой $C$-плотной компоненте $\Omega_{i}$ одномерного базисного множества $\Omega A$-диффеоморфизма $f$ ставится в соответствие автоморфизм группы $F$, определенньй с точностью до внутреннего автоморфизма.

Пару группа $F$ и автоморфизм $\tau_{i}$ группы $F$, определенный описанным выше образом $\left(\tau_{i}=\bar{f}_{i *}\right)$, будем обозначать через $\left(F, \tau_{i}\right)_{\Omega_{i}}$.

ОПРЕДЕЛЕНИЕ 3 . Пару $\left(F, \tau_{i}\right)_{\Omega_{i}}$ назовем алгебраическим представлением $C$-плотной компоненты $\Omega_{i}$ одномерного базисного множества $\Omega$.

Пусть $f, f^{\prime}$ структурно устойчивые диффеоморфизмы, неблуждающие множества которых содержат одномерные базисные множества $\Omega, \Omega^{\prime}$, состоящие из $m$ $C$-плотных компонент $\Omega_{1}, \ldots, \Omega_{m}, \Omega_{1}^{\prime}, \ldots, \Omega_{m}^{\prime}$, соответственно.

ОПРЕДЕЛЕНИЕ 4. Алгебраические представления $\left(F, \tau_{i}\right)_{\Omega_{i}},\left(F^{\prime}, \tau_{i}^{\prime}\right)_{\Omega_{i}^{\prime}}$ назовем алгебраически сопряженньми, если существует изоморфизм $\psi: F \rightarrow F^{\prime}$ такой, что $\tau_{i}^{\prime}=\psi \tau_{i} \psi^{-1}$.

ЛЕмма 5. Алгебрачческое представление $\left(F, \tau_{i}\right)_{\Omega_{i}}$ C-плотной компоненты одномерного базисного множества $\Omega$ диффеоморфизма $f$ есть инвариант топологической сопряженности с точностью до алгебрачческой сопряженности.

В работах [12], [13] установлено, что для того чтобы сушествовал гомеоморфизм $g: N_{\Omega} \rightarrow N_{\Omega^{\prime}}^{\prime}$ такой, что $g(\Omega)=\Omega^{\prime}$ и

$$
\left.f^{\prime}\right|_{\Omega}=\left.g f g^{-1}\right|_{\Omega^{\prime}}
$$

необходимо и достаточно, чтобы алгебраические представления $\left(F, \tau_{i}\right)_{\Omega_{i}},\left(F^{\prime}, \tau_{i}^{\prime}\right)_{\Omega_{i}^{\prime}}$ были алгебраически сопряжены при некоторой нумерации $C$-плотных компонент множеств $\Omega$ и $\Omega^{\prime}$ и некоторого $i \in\{1, \ldots, m\}$.

Напомним, что точка $x$, принадлежашая $W^{s}(p) \cap W^{u}(q)$, где $p, q(p \neq q)$ седловые периодические точки, называется гетероклинической точкой, а множество $G=\bigcup_{n \in \mathbb{Z}} f^{n}(x)$ - гетероклинической траекторией [20].

Пусть $p, q \in P_{1}(f)$ седловые периодические точки и $x \in L^{s}(p) \cap L^{u}(q)$ любая гетероклиническая точка, принадлежашая гетероклинической траектории $G=\bigcup_{n \in \mathbb{Z}} f^{n}(x)$, где $L^{s}(p), L^{u}(q)$ - компоненты связности множеств $W^{s}(p) \backslash p$, $W^{u}(q) \backslash q$, соответственно, содержашие точку $x$.

Обозначим через $m \in \mathbb{Z}^{+}$наименьшее число, для которого $f^{m}\left(L^{s}(p)\right)=L^{s}(p)$, $f^{m}\left(L^{u}(q)\right)=L^{u}(q)$. Зададим ориентацию на кривой $L^{s}(p)\left(L^{u}(q)\right)$ к точке $p$ 
(от точки $q$ ) и две нумерации множества точек $\left[x, f^{m}(x)\right)^{s} \cap L^{u}(q)$, первая из которых согласуется с ориентацией на $L^{s}(p)$, а вторая - на $L^{u}(q)$.

Обозначим через $S(x)$ подстановку, переводящую первую нумерацию во вторую.

ЛЕмма 6. Пусть у любая гетероклиническая точка, принадлежащая гетероклинической траектории $G=\bigcup_{n \in \mathbb{Z}} f^{n}(x)$, где $x \in L^{s}(p) \cap L^{u}(q), p, q \in P_{1}$, тогда $S(x)=S(y)$.

Из леммы 6 следует, что для каждой гетероклинической траектории $G$ корректно определяется подстановка $S(G)=S(x)$, где $x$ любая точка траектории $G$.

ОПРЕДЕЛЕНИЕ 5 . Подстановку $S(G)$ назовем гетероклинической подстановкой траектории $G$.

Лемма 7. Гетероклиническая подстановка есть инвариант топологической сопряасенности.

Зафиксируем ориентацию поверхности $M$ и выберем в точке $x \in G$ касательный вектор $\xi_{x}^{s}\left(\xi_{x}^{u}\right)$ к кривой $L^{s}(p)\left(L^{u}(q)\right)$, индуцирующий выбранное на ней направление.

Положим $J(x)=1(-1)$, если репер $\left\{\xi_{x}^{s}, \xi_{x}^{u}\right\}$ согласован (не согласован) с ориентацией $M$. Положим $J(G)=J(x)$. Так как диффеоморфизм $f$ сохраняет ориентацию многообразия $M$, число $J(G)$ не зависит от выбора точки $x$ на траектории $G$.

ОПРЕДЕЛЕНИЕ 6 . Число $J(G)$ назовем индексом гетероклинической траектории $G$.

Пусть $f, f^{\prime}$ топологически сопряженные диффеоморфизмы, посредством сопрягаюшего гомеоморфизма $g\left(f^{\prime}=g f g^{-1}\right), G^{\prime}=g(G)$, где $G, G^{\prime}$-гетероклинические траектории диффеоморфизмов $f, f^{\prime}$. Тогда $J\left(G^{\prime}\right)=\nu J(G)$, где $\nu=1(\nu=-1)$, если гомеоморфизм $g$ сохраняет (меняет) ориентацию многообразия $M$.

Обозначим через $\Gamma_{a}\left(\Gamma_{r}\right)$ множество всех граничных периодических точек, принадлежаших всем аттракторам (репеллерам) из $\Lambda$, и положим $\Gamma=\Gamma_{a} \cup \Gamma_{r}$. Пусть

$$
\begin{gathered}
W_{\Gamma_{a}}^{u}=\bigcup_{p \in \Gamma_{a}} W^{u}(p) \backslash p, \quad W_{\Gamma_{r}}^{s}=\bigcup_{p \in \Gamma_{r}} W^{s}(p) \backslash p, \quad W_{\varnothing \Gamma_{a}}^{s}=\bigcup_{p \in \Gamma_{a}} W_{\varnothing}^{s}(p), \\
W_{\varnothing \Gamma_{r}}^{u}=\bigcup_{p \in \Gamma_{r}} W_{\varnothing}^{u}(p), \quad W_{\Gamma}^{u}=W_{\Gamma_{a}}^{u} \cup W_{\varnothing \Gamma_{r}}^{u}, \quad W_{\Gamma}^{s}=W_{\Gamma_{r}}^{s} \cup W_{\varnothing \Gamma_{a}}^{s}, \\
W_{\Gamma}=W_{\Gamma}^{u} \cup W_{\Gamma}^{s}, \quad W_{P_{1}}^{\sigma}=\bigcup_{p \in P_{1}}\left(W^{\sigma}(p) \backslash p\right), \quad \sigma \in\{s, u\} .
\end{gathered}
$$

Обозначим через $\widehat{W}_{P_{1}}^{\sigma}$ подмножество множества $W_{P_{1}}^{\sigma}$, состоящее из точек, принадлежащих тем и только тем компонентам связности множества $W_{P_{1}}^{\sigma}$, которые содержат гетероклинические точки.

Положим

$$
\begin{gathered}
W_{P_{1}}=W_{P_{1}}^{s} \cup W_{P_{1}}^{u}, \quad \widehat{W}_{P_{1}}=\widehat{W}_{P_{1}}^{s} \cup \widehat{W}_{P_{1}}^{u}, \\
R=M \backslash\left(\Lambda \cup\left(W_{P_{1}} \backslash \widehat{W}_{P_{1}}\right) \cup W_{\Gamma} \cup P\right) .
\end{gathered}
$$


Пусть $Q$ некоторая компонента связности множества $R$. В силу лемм 1,2 и леммы 2 из [19] достижимая изнутри граница $\partial Q$ компоненты $Q$ является объединением двух одномерных комплексов

$$
\partial^{s} Q \subset\left(W^{s} \backslash \widehat{W}^{s}\right) \cup W_{\Gamma}^{s} \cup P, \quad \partial^{u} Q \subset\left(W^{u} \backslash \widehat{W}^{u}\right) \cup W_{\Gamma}^{u} \cup P .
$$

ОПРЕДЕЛЕНИЕ 7 . Компоненту связности множества $R$, содержашую гетероклинические точки, назовем гетероклинической областью.

Топологическая структура гетероклинических областей изучена в работе [19].

ОПРЕДЕЛЕНИЕ 8. Ориентированный граф $G(f)$ назовем графом диффеоморфизма $f$, если

I) вершины графа $G(f)$ соответствуют:

1) периодическим точкам из тривиальных базисных множеств,

2) граничным периодическим точкам из одномерных базисных множеств,

3) $C$-плотным компонентам одномерных базисных множеств,

4) гетероклиническим областям;

II) ребра графа $G(f)$ соответствуют компонентам связности множеств $W_{P_{1}}$ и $W_{\Gamma}$. При этом, если ребру $l^{\sigma}$ соответствует компонента связности $L^{\sigma}(p) \subset W_{P_{1}} \cup W_{\Gamma}$, то одна из двух вершин $a$ и $b$, инцидентных ребру $l^{\sigma}$, например, $a$ соответствует седловой периодической точке $p$, а для другой вершины $b$ имеет место в точности одна из следующих возможностей:

1 ) если точка $p \in \Gamma_{a}$ и $\sigma=u$, то вершина $b$ соответствует $C$-плотной компоненте аттрактора, содержашего точку $p$, совпадающей с множеством $\partial L^{\sigma}(p)$, если $p \in \Gamma_{a}$ и $\sigma=s$, то точка $b$ соответствует источниковой периодической точке, совпадаюшей с множеством $\partial L^{\sigma}(p)$;

2 ) если точка $p \in \Gamma_{r}$ и $\sigma=s$, то вершина $b$ соответствует $C$-плотной компоненте репеллера, содержащего точку $p$, совпадающей с множеством $\partial L^{\sigma}(p)$, если $p \in \Gamma_{r}$ и $\sigma=u$, то точка $b$ соответствует стоковой периодической точке, совпадающей с множеством $\partial L^{\sigma}(p)$;

3 ) если точка $p \in P_{1}, L^{\sigma}(p) \subset M_{\Lambda}$ и $\sigma=s(\sigma=u)$, то вершина $b$ соответствует $C$-плотной компоненте некоторого репеллера (аттрактора), совпадаюшей с множеством $\partial L^{\sigma}(p)$;

4) если точка $p \in P_{1}, L^{\sigma}(p)$ принадлежит некоторой гетероклинической компоненте $Q$ диффеоморфизма $f$, то вершина $b$ соответствует $Q$;

$5)$ если точка $p \in P_{1}$, а $L^{\sigma}(p)$ не принадлежит $M_{\Lambda}$, не принадлежит никакой гетероклинической области и $\sigma=s(\sigma=u)$, то вершина $b$ соответствует источниковой (стоковой) периодической точке, совпадающей с множеством $\partial L^{\sigma}(p)$.

Ориентация на ребре $l$ выбирается от $b$ к $a$, если $\sigma=s$, и от $a$ к $b$, если $\sigma=u$.

Подмножества графа $G(f)$, соответствуюшие достижимьм изнутри границам компонент связности из множества $R$, назовем различающими множествами. 
Для вершины $b$, соответствуюшей периодической точке $p \in P$ из тривиального базисного множества диффеоморфизма $f$, обозначим через $U(b)$ множество всех ребер графа $G(f)$, инцидентных вершине $b$, и обозначим через $N(b)$ мощность множества $U(b)$ (если вершина $b$ соответствует седловой периодической точке, то $N(b)=4)$. Зададим нумерацию в множестве ребер $U(b)$ следуюшим образом. Выберем в окрестности точки $p$ простую гладкую замкнутую кривую $L$ такую, что кривая $L$ ограничивает диск $D$, содержаший точку $p$, и пересекается в точности в одной точке с каждой компонентой связности $L^{\sigma}(q) \notin \widehat{W}_{P_{1}}^{\sigma}$ множества $W^{\sigma}(q) \backslash q$, $q \in P_{1}, p \in \bar{L}^{\sigma}(q)$ (если точка $p$ седловая, то $q=p$ ).

Зададим в некоторой точке кривой $L$ репер $(\tau, n)$, согласованный с ориентацией многообразия $M$, такой, что вектор $\tau$ касается кривой $L$ и вектор $n$ направлен внутрь диска $D$. Зафиксируем направление обхода по кривой $L$, индуцированное вектором $\tau$. Занумеруем компоненты $L_{1}, \ldots, L_{N(b)}$ в порядке, в котором они встречаются при обходе вдоль $L$, начиная с некоторой точки на $L$, и снабдим соответствующие им ребра из $U(b)$ теми же номерами. Будем называть полученную нумерацию положительно ориентированной. Нумерацию, которая получается при противоположном обходе, назовем отрицательно ориентированной.

Для вершины $\gamma$, отвечаюшей гетероклинической области $Q$, обозначим через $Q^{s}(\gamma)\left(Q^{u}(\gamma)\right)$ множество всех ребер, для которых $\gamma$ является началом (концом). Обозначим через $r$ минимальное положительное число, для которого все точки из тривиальных базисных множеств неподвижны относительно $f^{r}$ и компоненты связности множества $W^{\sigma}(p) \backslash p$ инвариантны относительно $f^{r}$ для любой точки $p \in P_{1}$.

Для каждого ребра $l^{\sigma} \in Q^{\sigma}(\gamma)$ обозначим через $L^{\sigma}$ соответствующую ему компоненту и через $N\left(l^{\sigma}\right)$ - число всех гетероклинических траекторий диффеоморфизма $f^{r}$, имеющих с кривой $L^{\sigma}$ непустое пересечение.

Пусть $x_{1}^{\sigma}, \ldots, x_{N\left(l^{\sigma}\right)}^{\sigma}$ гетероклинические точки на кривой $L^{\sigma}$, занумерованные в соответствии с ориентацией на ней, выбранные таким образом, что на дуге $\left[x_{1}^{\sigma}, x_{N\left(l^{\sigma}\right)}^{\sigma}\right] \subset L^{\sigma}$ нетгетероклинических точек, отличных от них, ипринадлежащие по одной всем различнњм гетероклиническим траекториям диффеоморфизма $f^{r}$, имеющим с $L$ непустое пересечение.

Определим отображение $\varphi_{\gamma}^{l^{\sigma}}$ из множества $\left\{1, \ldots, N\left(l^{\sigma}\right)\right\}$ в множество $Q^{\bar{\sigma}}(\gamma)$, полагая $\varphi_{\gamma}^{l^{\sigma}}(j)=l^{\bar{\sigma}}$, где $l^{\bar{\sigma}}$ является ребром, для которого $L^{\bar{\sigma}} \cap L^{\sigma}=x_{j}$.

Определим отображение $\psi_{\gamma}^{l^{\sigma}}$ из множества $\left\{1, \ldots, N\left(l^{\sigma}\right)\right\}$ в множество подстановок, полагая $\psi_{\gamma}^{l^{\sigma}}(j)=S\left(x_{j}\right)$ (подстановка $S\left(x_{j}\right)$ определяется для диффеоморфизма $f^{r}$ ).

Определим отображение $\phi_{\gamma}^{l^{\sigma}}$ из множества $\left\{1, \ldots, N\left(l^{\sigma}\right)\right\}$ в множество $\{-1,1\}$, полагая $\phi_{\gamma}^{l^{\sigma}}(j)=J\left(x_{j}\right)$.

ОПРЕДЕЛЕНИЕ 9. Назовем различающим графом $G^{*}(f)$ граф $G(f)$, оснашенньй:

1) набором различающих множеств;

2) весами седловых вершпн ${ }^{5}$;

\footnotetext{
${ }^{5}$ Вершине $a$, отвечающей седловой периодической точке $p$ периода $k$, припишем вес +1
} 
3) положительно или отрицательно ориентированньми нумерациями в множествах $U(b)$ одновременно для всех вершин $b$, соответствующих периодическим точкам из тривиальных базисных множеств;

4) числами $N\left(l^{\sigma}\right)$ и отображениями $\varphi_{\gamma}^{l^{\sigma}}, \psi_{\gamma}^{l^{\sigma}}, \phi_{\gamma}^{l^{\sigma}}, \sigma \in\{s, u\}$, где $\gamma$ соответствует гетероклинической области, $l^{\sigma} \in Q^{\sigma}(\gamma)$;

5) алгебраическими представлениями для вершин, соответствующих $C$-плотным компонентам аттракторов и репеллеров.

Пусть теперь имеются два структурно устойчивых диффеоморфизма $f, f^{\prime}$ из рассматриваемого класса $S(M)$ с различающими графами $G^{*}(f), G^{*}\left(f^{\prime}\right)$, соответственно.

Предположим, что неблуждающие множества диффеоморфизмов $f, f^{\prime}$ содержат одномерные базисные множества $\Omega, \Omega^{\prime}$, соответственно, состоящие из $m C$-плотных компонент $\Omega_{1}, \ldots, \Omega_{m}, \Omega_{1}^{\prime}, \ldots, \Omega_{m}^{\prime}$. Пусть $w_{i}\left(w_{i}^{\prime}\right)$ вершины графа $G(f)\left(G^{\prime}\left(f^{\prime}\right)\right)$, соответствующие компонентам $\Omega_{i}\left(\Omega_{i}^{\prime}\right), i=\overline{1, m}$. Обозначим через $B\left(w_{i}\right)\left(B^{\prime}\left(w_{i}^{\prime}\right)\right)$ множество всех вершин графа $G^{*}(f)\left(G^{\prime *}\left(f^{\prime}\right)\right)$, соответствующих граничным периодическим точкам, принадлежащим $C$-плотной компоненте $\Omega_{i}\left(\Omega_{i}^{\prime}\right)$, и через $R\left(w_{i}\right)\left(R^{\prime}\left(w_{i}^{\prime}\right)\right)$ множество всех ребер графа $G^{*}(f)\left(G^{*}\left(f^{\prime}\right)\right)$, соединяющих вершину $w_{i}\left(w_{i}^{\prime}\right)$ с вершинами из множества $B\left(w_{i}\right)\left(B^{\prime}\left(w_{i}^{\prime}\right)\right)$.

ЛЕмма 8. Пусть алгебраические представления $\left(F, \tau_{i}\right)_{\Omega_{i}},\left(F^{\prime}, \tau_{i}^{\prime}\right)_{\Omega_{i}^{\prime}}$ алгебраически сопряжены в силу некоторого изоморфизма $\psi$. Тогда автоморфизм $\psi$ индуиирует единственным образом взаимно однозначное отображсение $\psi_{*}$ множества $B\left(w_{i}\right) \cup R\left(w_{i}\right)$ на множество $B^{\prime}\left(w_{i}^{\prime}\right) \cup R^{\prime}\left(w_{i}^{\prime}\right)$.

ОПРЕДЕЛЕНИЕ 10. Назовем различающие графы $G^{*}(f), G^{*}\left(f^{\prime}\right)$ диффеоморфизмов $f, f^{\prime}$ изоморфнылми, если сушествует изоморфизм $\eta$ графов $G^{*}(f), G^{*}\left(f^{\prime}\right)$ такой, что

1) $\eta$ преобразует вершины, соответствующие стоковым, источниковым, седловьм периодическим точкам из тривиальных базисных множеств, граничным периодическим точкам из одномерных базисных множеств, $C$-плотным компонентам одномерных базисных множеств и гетероклиническим областям графа $G^{*}(f)$, в соответствующие вершины граффа $G^{*}\left(f^{\prime}\right)$;

2) $\eta$ сохраняет ориентацию ребер;

3) $\eta$ преобразует различающие множества графа $G^{*}(f)$ в различающие множества графа $G^{\prime *}\left(f^{\prime}\right)$;

4) $\eta$ сохраняет веса вершин, отвечающих седловым периодическим точкам из тривиальных базисных множеств;

$5)$ алгебраические представления $\left(F, \tau_{i}\right),\left(F^{\prime}, \tau_{i}^{\prime}\right)$, которьми оснашены изомофные в силу $\eta$ вершины $w_{i}, w_{i}^{\prime}=\eta\left(w_{i}\right)$, отвечающие $C$-плотным компонентам $\Omega_{i}, \Omega_{i}^{\prime}$ одномерных аттракторов (репеллеров) $\Omega, \Omega^{\prime}$, алгебраически

$(-1)$, если отображение $D f_{p}^{k}: E_{p}^{u} \rightarrow E_{p}^{u}$ сохраняет (меняет) ориентацию $E_{p}^{u}$. Здесь $E_{p}^{u}-$ инвариантное относительн $D f_{p}^{k}$ одномерное расширяющееся подпространство касателннго пространства в точке $p$. 
сопряжены; при этом, если $\psi$ - сопрягающий изоморфизм и $\psi_{*}-$ индуцированное отображение $B\left(w_{i}\right) \cup R\left(w_{i}\right)$ на $B^{\prime}\left(w_{i}^{\prime}\right) \cup R\left(w_{i}^{\prime}\right)$, то ограничение изоморфизма $\eta$ на множество $B\left(w_{i}\right) \cup R\left(w_{i}\right)$ совпадает с отображением $\psi_{*}$;

6) $\eta$ сохраняет номера изоморфных ребер в множествах $U(b), U^{\prime}\left(b^{\prime}\right)$, где $b^{\prime}=\eta(b)$;

7) если $\gamma^{\prime}=\eta(\gamma)$ и $l^{\prime \sigma}=\eta\left(l^{\sigma}\right), l^{\sigma} \in Q^{\sigma}(\gamma), l^{\prime \sigma} \in Q^{\prime \sigma}\left(\gamma^{\prime}\right)$, тогда $N\left(l^{\sigma}\right)=$ $N^{\prime}\left(l^{\prime \sigma}\right), \eta\left(\varphi_{\gamma}^{l^{\sigma}}\right)=\varphi_{\gamma^{\prime}}^{\prime l^{\prime \sigma}}, \eta\left(\psi_{\gamma}^{l^{\sigma}}\right)=\psi_{\gamma^{\prime}}^{\prime l^{\prime \sigma}}, \phi_{\gamma}^{l^{\sigma}}=\nu \phi_{\gamma^{\prime}}^{\prime l^{\prime \sigma}}$, где $\nu=1(\nu=-1)$, если ориентации в нумерациях множеств $U(b), U^{\prime}\left(b^{\prime}\right)$ совпадают (противоположны).

ЛЕмма 9. Различающий граф $G^{*}(f)$ есть инвариант топологической сопряхсенности с точностью до изоморфизма.

Диффеоморфизмы $f, f^{\prime}$ индуцируют на множествах вершин графов $G^{*}(f)$, $G^{*}(f)$ подстановки $P(f), P^{\prime}\left(f^{\prime}\right)$.

ТЕОРема 2. Для того чтобы структурно устойчивые диффеоморфизмы $f, f^{\prime} \in S(M)$ были топологически сопряэсены необходимо и достаточно, чтобы существовал изоморфизм $\eta$ графов $G^{*}(f), G^{* *}(f)$ такой, что $P^{\prime}\left(f^{\prime}\right)=$ $\eta P(f) \eta^{-1}$.

\section{§ 2. Построение канонических форм одномерных аттракторов и репеллеров}

Этот параграф̆ посвяшен доказательству лемм 1-4. Для простоты изложения мы докажем утверждения этих лемм в предположении, что одномерное базисное множество $\Omega$ есть аттрактор (в случае если $\Omega$ является репеллером, то для доказательства достаточно рассмотреть диффеоморфизм $\left.f^{-1}\right)$.

ДокАЗАТЕЛЬство лЕммы 1. Докажем вначале, что для любой точки $z$, принадлежащей нетривиальному базисному множеству диффеоморфизма $f, W^{u}(z) \cap$ $(x, y)^{s}=\varnothing$. Предположим противное, пусть существуют нетривиальное базисное множество $\Omega^{\prime}$ и точка $z \in \Omega^{\prime}$ такие, что $W^{u}(z) \cap(x, y)^{s} \neq \varnothing$ (по предположению $\left.\Omega^{\prime} \neq \Omega\right)$. Возможны два случая:

1) многообразие $W^{u}(z)$ не содержит граничной периодической точки базисного множества $\Omega^{\prime}$;

2) многообразие $W^{u}(z)$ содержит граничную периодическую точку типа $u$ или типа $(s, u)$ базисного множества $\Omega^{\prime}$.

В случае 1$)$ обозначим через $L(x)(L(y))$ компоненту связности множества $W^{u}(x) \backslash x\left(W^{u}(y) \backslash y\right)$, не содержашую точку $p(q)$. Положим $l_{x y}=L(x) \cup L(y) \cup$ $[x, y]^{s}$. В силу [9], [11], [33] кривая $l_{x y}$ ограничивает область $D_{x y}$, гомеоморфную диску, состоящую из блуждающих точек диффеоморфизма $f$, для которой кривая $l_{x y}$ является достижимой изнутри гранищей.

Так как дуга $(x, y)^{s}$ состоит из блуждающих точек диффеоморфизма $f$, то точка $z \notin(x, y)^{s}$. Тогда сушествует точка $z_{1}$, принадлежашая $W^{u}(z) \cap(x, y)^{s}$, такая, что $\left(z, z_{1}\right)^{u} \cap(x, y)^{s}=\varnothing$. Обозначим через $W_{*}^{u}\left(z_{1}\right)$ компоненту связности множества $W^{u}\left(z_{1}\right) \backslash z_{1}$, не содержащую точку $z$. В силу строгого условия трансверсальности 
$W_{*}^{u}\left(z_{1}\right) \cap D_{x y} \neq \varnothing$. В силу $[9]$ компонента $W_{*}^{u}\left(z_{1}\right)$ содержит множество, плотное в $C$-плотной компоненте множества $\Omega^{\prime}$. Поэтому на компоненте $W_{*}\left(z_{1}\right)$ существуют точки, не принадлежащие области $D_{x y}$. Но тогда найдется точка $z_{2} \in(x, y)^{s}$, отличная от точки $z_{1}$, такая, что дуга $\left(z_{1}, z_{2}\right)^{u}$ целиком принадлежит области $D_{x y}$. Так как для любой точки $\widetilde{x} \in W_{x}^{u}(p)$ найдется единственная точка $\widetilde{y} \in W_{y}^{u}(q)$ такая, что $\widetilde{y} \in W^{s}(\widetilde{x})$ и $(\widetilde{x}, \widetilde{y})^{s} \subset D_{x y}$, то существует точка $\widetilde{x}$, для которой дуга $\Delta^{s}(\widetilde{x})$ касается дуги $\left(z_{1}, z_{2}\right)^{u}$ в некоторой точке $\widetilde{z}$, что противоречит строгому условию трансверсальности (рис. 5).

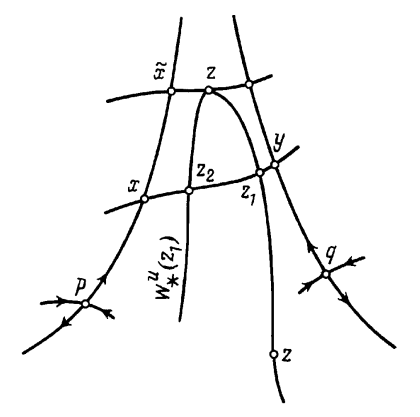

Рис. 5

Рассмотрим случай 2). Так как периодические точки плотны в множестве $\Omega^{\prime}$ и множество граничных периодических точек конечно, то в сколь угодно малой окрестности точки $z$ найдется внутренняя периодическая точка множества $\Omega^{\prime}$. Но тогда в силу $C^{1}$-близости неустойчивых многообразий точек из $\Omega^{\prime}$ на компактных множествах следует, что сушествует внутренняя периодическая точка $z^{\prime} \in \Omega^{\prime}$ такая, что $W^{u}\left(z^{\prime}\right) \cap(x, y)^{s} \neq \varnothing$, и мы приходим к случаю 1$)$.

Далее могут быть два случая:

a) дуга $(x, y)^{s}$ не содержит точек, принадлежащих неустойчивым многообразиям седловых периодических точек из тривиальных базисных множеств;

б) дуга $(x, y)^{s}$ содержит точки, принадлежащие неустойчивым многообразиям седловых периодических точек из тривиальных базисных множеств.

В случае а) покажем, что компонента $W_{\varnothing}^{s}(p)$ целиком принадлежит неустойчивому многообразию в точности одной источниковой периодической точки $\alpha_{1}$ диффеоморфизма $f$, и следовательно, $\partial W_{\varnothing}^{s}(p)=\alpha_{1}$. Предположим противное. В силу [20] многообразие $M$ представляется в виде объединения неустойчивых многообразий базисных множеств. Тогда из условия строгой трансверсальности, из всюду плотности множества периодических точек в неблуждающем множестве и из того, что неустойчивое многообразие периодической источниковой точки гомеоморфно открытому диску, следует, что сушествует седловая периодическая точка $p^{\prime}$ такая, что $W^{u}\left(p^{\prime}\right) \cap W_{\varnothing}^{s}(p) \neq \varnothing$. Но тогда в силу условия строгой трансверсальности и в силу [20] $W^{u}\left(p^{\prime}\right) \cap(x, y)^{s} \neq \varnothing$, что противоречит предположению случая а). Если $p \neq q$, то совершенно аналогично устанавливается, что компонента $W_{\varnothing}^{s}(q)$ целиком принадлежит неустойчивому многообразию в точности одной источниковой периодической точки $\beta$, и следовательно, $\partial W_{\varnothing}^{s}(p)=\beta$. Покажем, 
что $\alpha_{1}=\beta$. Предположим противное. Тогда на дуге $(x, y)^{s}$ сушествуют точки, принадлежащие неустойчивым многообразиям точек $\alpha_{1}$ и $\beta$, и следовательно, на ней должна существовать точка, принадлежащая неустойчивому многообразию некоторой седловой периодической точки, что противоречит предположению. По построению множество $L_{p q}=W_{\varnothing}^{s}(p) \cup W_{\varnothing}^{s}(q) \cup \alpha_{1} \cup p \cup q$ является связньгм одномерным комплексом, удовлетворяющим требованиям 1)-5) леммы 1 в случае $n=0$ (рис. 3a).

В случае б) пусть $p$ седловая периодическая точка, принадлежащая тривиальному базисному множеству, для которой многообразие $W^{u}(p)$ пересекается с дугой $(x, y)^{s}$ в некоторой точке $z \in(x, y)^{s}$. Как и в случае а) устанавливается, что компонента связности $W_{z}(p)$ множества $W^{u}(p) \backslash p$, содержащая точку $z$, пересекается с дугой $(x, y)^{s}$ в точности в одной точке $z$. Поэтому сушествует конечное число различных седловых периодических точек $p_{1}, \ldots, p_{n}, n \geqslant 1$ (принадлежаших тривиальным базисньм множествам), для которых $W^{u}\left(p_{i}\right) \cap(x, y)^{s} \neq \varnothing$, $i=\overline{1, n}$. Обозначим через $z_{1}, \ldots, z_{r}, r \leqslant 2 n$, все точки, принадлежащие пересечению $\bigcup_{i=1}^{n}\left(W^{u}\left(p_{i}\right) \cap(x, y)^{s}\right)$ и занумерованные в соответствии с ориентацией на дуге $(x, y)^{s}$, выбранной в направлении от точки $x$ к точке $y$ (см. рис. 3б). Для точки $z_{i}$ обозначим через $p_{n_{i}}, n_{i} \in\{1, n\}$, периодическую точку, неустойчивое многообразие которой содержит точку $z_{i}$. Предположим, что при движении от точки $x \mathrm{k}$ точке $y$ по дуге $(x, y)^{s}$ при переходе через точку $z_{i}$ дуга $\left(p_{n_{i}}, z_{i}\right)^{u} \subset W^{u}\left(p_{i}\right)$ остается справа. Тогда обозначим через $W_{-}^{s}\left(p_{i}\right), W_{+}^{s}\left(p_{i}\right)$ те компоненты связности множества $W^{s}\left(p_{i}\right) \backslash p_{i}$, что если на компоненте $W_{-}^{s}\left(p_{i}\right)$ выбрать произвольную точку $z_{i}^{-}$, а на компоненте $W_{+}^{s}\left(p_{i}\right)$ - произвольную точку $z_{i}^{+}$, то при движении по дуге $\left(z_{i}^{-}, z_{i}^{+}\right)^{s} \subset W^{s}\left(p_{i}\right)$ при переходе через точку $p_{i}$ дуга $\left(p_{n_{i}}, z_{i}\right)^{u} \subset W^{u}\left(p_{i}\right)$ остается слева.

Так же как и в случае а) устанавливаем, что компоненты $W_{\varnothing}^{s}(p)\left(W_{\varnothing}^{s}(q)\right)$ и $W_{-}^{s}\left(p_{n_{1}}\right)\left(W_{+}^{s}\left(p_{n_{r}}\right)\right)$ принадлежат неустойчивому многообразию некоторой источниковой периодической точки $\beta_{1}\left(\beta_{r+1}\right)$ (если $p=q$, то $\left.\beta_{1}=\beta_{r+1}\right)$. Компоненты $W_{+}^{s}\left(p_{n_{i}}\right)$ и $W_{-}^{s}\left(p_{n_{i+1}}\right), i=\overline{1, r-1}$, принадлежат неустойчивому многообразию некоторой источниковой периодической точки $\beta_{i+1}$ (если точки $z_{i}, z_{i+1}$ принадлежат неустойчивому многообразию одной и той же седловой периодической точки, т.е. $p_{n_{i}}=p_{n_{i+1}}$, то $\left.W_{+}^{s}\left(p_{n_{i}}\right)=W_{-}^{s}\left(p_{n_{i+1}}\right)\right)$.

Обозначим через $l \geqslant 1$ число различных источниковых точек среди множества $\left\{\beta_{1}, \ldots, \beta_{r+1}\right\}$ и переобозначим их через $\alpha_{1}^{u}, \ldots, \alpha_{l}^{u}$. Тогда каждое из множеств $\partial W_{\varnothing}^{s}(p), \partial W_{\varnothing}^{s}(q)$ состоит в точности из одной источниковой периодической точки, принадлежащей $\left\{\alpha_{i}^{u}\right\}$, а каждое из множеств $\partial W^{s}\left(p_{i}\right), i \in\{1, n\}$, состоит в точности из одной или двух источниковых точек, принадлежащих множеству $\left\{\alpha_{i}^{u}\right\}$, $i \in\{1, \ldots, l\}$. Тогда множество

$$
L_{p q}=W_{\varnothing}^{s}(p) \cup W_{\varnothing}^{s}(q) \cup \bigcup_{i=1}^{n} W^{s}\left(p_{i}\right) \cup \bigcup_{i=1}^{l} \alpha_{i} \cup p \cup q
$$

является связным одномерным комплексом, удовлетворяющим требованиям 1)-5) леммы 1 в случае $n>0$ (рис. 3б). Лемма 1 полностью доказана. 
ДОКАЗАТЕЛЬСТВо ЛЕМмЫ 2. Пусть $C=W^{u}\left(p_{1}\right) \cup \cdots \cup W^{u}\left(p_{r}\right)$ связка степени $r$ аттрактора $\Omega$ и $N \in \mathbb{Z}^{+}$число, для которого компоненты связности множеств $W^{u}\left(p_{i}\right) \backslash p_{i}$ инвариантны относительно $f^{N}$ для всех $i=\overline{1, r}$. Положим

$$
L(C)=\bigcup_{i=1}^{r}\left(x_{2 i-1}, x_{2 i}\right)^{u} \cup\left(x_{2 i}, x_{2 i+1}\right)^{s}
$$

(здесь $x_{1}, \ldots, x_{2 r}$ - последовательность точек, входящая в определение связки $C$ ). Положим $L_{n}(C)=f^{-n N}(L), n \in \mathbb{Z}^{+}$, и обозначим через $L_{*}(C)$ топологический предел при $n \rightarrow \infty$ последовательности кривых $L_{n}(C)$.

По доказанному в лемме 1 топологический предел последовательности дуг $f^{-k N}\left(\left(x_{2 i}, x_{2 i+1}\right)^{s}\right)$ есть одномерный комплекс $l_{p_{i} p_{i+1}}$, обладающий свойствами 1)-5).

Но тогда $L_{*}(C)$ есть связный комплекс, состоящий из граничных периодических точек $p_{1}, \ldots, p_{r}$, компонент связности $W_{\varnothing}^{s}\left(p_{i}\right), i=\overline{1, r}$, источниковых периодических точек и устойчивых многообразий седловых периодических точек, принадлежащих тривиальным базисным множествам диффеоморфизма $f$. Обозначим через $\widehat{C}_{i}$ множество всех связок $C$, принадлежащих $i$-й $C$-плотной компоненте. Положим $B_{i}=\bigcup_{C \in \widehat{C}_{i}} L_{*}(C)$. По построению множество $B_{i}$ является границей открытого, инвариантного относительно $f^{m}$ подмножества $M_{i}\left(f^{m}\left(M_{i}\right)=M_{i}\right)$ такого, что $\Omega_{i} \backslash \Gamma_{i} \subset M_{i}$ и $M_{i} \backslash \Omega_{i}$ не содержит точек неблуждающего множества диффеоморфизма $f$. Тогда множество $M_{\Omega}$, являюшееся объединением $m$ открытых подмножеств $M_{1}, \ldots, M_{m}$, удовлетворяет всем требованиям леммы 2 . Лемма 2 доказана.

ДоКАЗАТЕЛЬСТво ЛЕмМЫ 3. Рассмотрим для определенности случай $\sigma=u$ (если $\sigma=s$, то доказательство аналогичное). Докажем первое утверждение леммы 3. Пусть $L^{u}(p) \cap M_{\Lambda} \neq \varnothing$, тогда существует аттрактор $\Omega$ такой, что $L^{u}(p) \cap M_{\Omega} \neq \varnothing$. В силу леммы 1 и конструкции множества $M_{\Omega}$ в лемме 2 следует, что существуют граничные периодические точки $p_{1}, p_{2}$ ( $p_{1}$ может совпадать с $\left.p_{2}\right)$ аттрактора $\Omega$ и область $D_{p_{1} p_{2} x y}$, построенная в лемме 1 , такие, что $L^{u}(p) \cap D_{p_{1} p_{2} x y} \neq \varnothing\left(x \in W^{u}\left(p_{1}\right), y \in W^{u}\left(p_{2}\right)\right.$, если $p_{1}=p_{2}$, то $x, y$ принадлежат различным компонентам связности множества $\left.W^{u}\left(p_{1}\right) \backslash p_{1}\right)$.

Пусть $N \in \mathbb{Z}^{+}$такое, что компонента $W^{u}(p)$ и компоненты $W_{x}^{u}\left(p_{1}\right), W_{y}^{u}\left(p_{2}\right)$, входящие в достижимую границу области $D_{p_{1} p_{2} x y}$, инвариантны относительно диффеоморфизма $f^{N}$.

Рассмотрим любую точку $z \in L^{u}(p) \cap D$ и положим $\lambda=\left[z, f^{N}(z)\right]$. Тогда $L^{u}(p)=\bigcup_{n \in \mathbb{Z}} f^{n N}(\lambda)$. Для любой точки $\widetilde{z} \in \lambda$ сушествуют точки $z_{1} \in W_{x}^{u}$, $z_{2} \in W_{y}^{u}$ такие, что $\widetilde{z} \in\left(z_{1}, z_{2}\right)^{s}$. Так как последовательность точек $f^{n N}(z)$ стремится к точке $p$ при $n \rightarrow-\infty$, а расстояние между точками $f^{n N}(z)$ и $f^{n N}\left(z_{1}\right)$ $\left(f^{n N}(z)\right.$ и $\left.f^{n N}\left(z_{2}\right)\right)$ стремится к нулю при $n \rightarrow+\infty$, то получаем, что точка $p$ принадлежит границе множества $M_{\Lambda}$, а множество $\partial L^{u}(p)$ состоит из $C$-плотной компоненты аттрактора $\Omega$, содержащей точки $p_{1}, p_{2}$. Таким образом, утверждение 1) леммы 3 доказано.

Предположим, что $L^{u}(p) \cap M_{\Lambda}=\varnothing$ и $L^{u}(p)$ не содержит гетероклинических точек. Тогда как и в лемме 1 устанавливается, что $\partial L^{u}(p)$ состоит из единственной стоковой периодической точки, т.е. имеет место второе утверждение леммы 3. 
Предположим, наконец, что $L^{u}(p) \cap M_{\Lambda}=\varnothing$ и $L^{u}(p)$ содержит гетероклинические точки, тогда рассмотрим множество $Q$, состояшее из всех седловых периодических точек таких, что если $q \in Q$, то $W^{s}(q) \cap L^{u}(p) \neq \varnothing$.

Множество $Q$ не может содержать точек из одномерного базисного множества, так как в противном случае компонента $L^{u}(p)$ имела бы непустое пересечение с множеством $M_{\Lambda}$, что противоречит предположению. В силу условия 2 ), определяющего рассматриваемый нами класс диффеоморфизмов, множество гетероклинических точек, принадлежащих пересечению устойчивых и неустойчивых многообразий седловых периодических точек из тривиальных базисных множеств, конечно. Поэтому для любой точки $q \in Q$ множество $W^{u}(q)$ не содержит гетероклинических точек, и следовательно, множество $\overline{W^{u}(q)} \backslash W^{u}(q)$ состоит из не более чем двух стоковых периодических точек диффеоморфизма $f$. Таким образом, множество $\bigcup_{q \in Q} W^{u}(q)$ есть связный одномерный комплекс, состоящий из стоковых периодических точек и неустойчивых многообразий седловых периодических точек, принадлежащих тривиальным базисньм множествам диффеоморфизма $f$. Лемма 3 доказана.

ДокАЗАТЕЛЬСТво ЛЕМмы 4. Для каждой кривой

$$
L(C)=\bigcup_{i=1}^{r}\left(x_{2 i-1}, x_{2 i}\right)^{u} \cup\left(x_{2 i}, x_{2 i+1}\right)^{s},
$$

рассмотренной в лемме 2 , построим в ее окрестности простую замкнутую кривую $\widetilde{L}(C)$ следующим образом. Для каждого $i, i=\overline{1, r}$, выберем пару точек $\widetilde{x}_{2 i-1}, \widetilde{x}_{2 i}$ и простую кривую $l_{i}$ с граничными точками $\widetilde{x}_{2 i-1}, \widetilde{x}_{2 i}$ такие, что:

1) $\widetilde{x}_{2 i-1} \in W^{s}\left(x_{2 i-1}\right), \widetilde{x}_{2 i} \in W^{s}\left(x_{2 i}\right)$;

2) $\left(\widetilde{x}_{2 i}, \widetilde{x}_{2 i+1}\right)^{s} \subset\left(x_{2 i}, x_{2 i+1}\right)^{s}\left(x_{2 r+1}=x_{1}\right)$;

3) кривая $l_{i}$ трансверсально пересекается с устойчивым многообразием любой точки, принадлежащей дуге $\left(x_{2 i-1}, x_{2 i}\right)^{u}$, в точности в одной точке;

4) кривая $\widetilde{L}(C)=\bigcup_{i=1}^{r} l_{i} \cup\left(\widetilde{x}_{2 i}, \widetilde{x}_{2 i+1}\right)^{s}$ является простой замкнутой кривой такой, что $\widetilde{L}(C), f(\widetilde{L}(C)), f^{-1}(\widetilde{L}(C))$ принадлежат $\bar{M}_{i} \backslash \Omega$;

$5)$ для каждой связки $C$ множества $\Omega$ найдется связка $C^{\prime}$ такая, что $f\left(\widetilde{L}\left(C^{\prime}\right)\right) \cap$ $\widetilde{L}(C)=\varnothing$ и кривые $\widetilde{L}(C), f\left(\widetilde{L}\left(C^{\prime}\right)\right)$ ограничивают область $K(C)$, гомеоморфную открытому кольцу и принадлежащую области, достижимая изнутри граница которой состоит из связки $C$ и кривой $\widetilde{L}(C)$ (рис. 6 ).

Положим

$$
\widehat{C}=\bigcup_{i=1}^{m} \widehat{C}_{i}, \quad V=\bigcup_{n \in \mathbb{Z}^{+}, C \in \widehat{C}} f^{n}(\overline{K(C)}) \cup \Omega .
$$

Тогда по построению множество $V$ является замкнутой окрестностью множества $\Omega$ и представляется в виде объединения $V=\bigcup_{i=1}^{m} V_{i}$ непересекающихся между собой областей $V_{i}$, являюшихся замкнутыми окрестностями множеств $\Omega_{i}$. Обозначим $V_{*}=\bigcup_{C \in \widehat{C}} \overline{K(C)} \cup f^{-1}(\overline{K(C)})$. Множество $V_{*}$ представляется в виде конечного объединения $V_{*}=\bigcup_{i=1}^{m} V_{* i}$ непересекающихся замкнутых подмножеств 


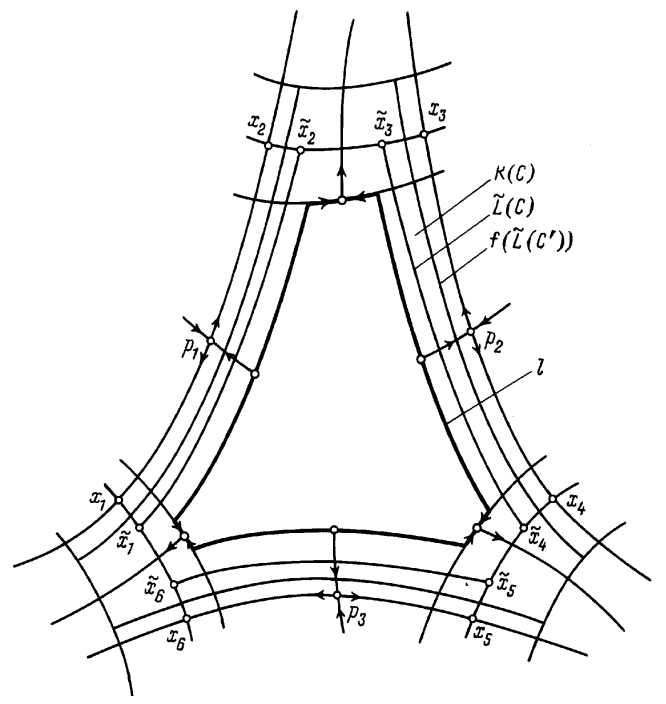

Рис. 6

$V_{* i}$ таких, что множество $N_{i}=V_{i} \cup V_{* i}$ является связным компактным двумерным многообразием с краем, содержашим компоненту $\Omega_{i}$.

Положим $N_{\Omega}=\bigcup_{i=1}^{m} N_{i}$. Тогда по построению множество $N_{\Omega}$ является компактным двумерным многообразием с краем, являюшимся объединением $m$ компактных двумерных многообразий с краем $N_{i}$, причем имеют место следующие свойства:

a) $\Omega \in N_{\Omega}$;

б) $\Omega_{i} \subset V_{i} \subset N_{i} \subset \bar{M}_{i}, \partial N=\bigcup_{i=1}^{m} \partial N_{i}$;

в) число компонент края подмногообразия $N_{i}$ равно $R(\Omega)$, число компонент края подмногообразия $N_{\Omega}$ равно $m R(\Omega)$.

Положим $V_{*}^{\prime}=V_{*} \cup\left(\bigcup_{C \in \widehat{C}} f(\overline{K(C)})\right)$. Тогда $\partial N_{\Omega}$ является частью гранищы множества $V_{*}^{\prime}$, и для каждой компоненты $l$ границы $\partial N_{\Omega}$ найдется в точности одна связка $C(l) \in \widehat{C}$ такая, что $l$ и $C(l)$ образуют достижимую изнутри границу области, гомеоморфную открытому кольцу и принадлежашую множеству $N_{\Omega} \backslash \Omega$ (компонента $l$ принадлежит множеству $\bigcup_{C \in \widehat{C}} f^{-1}(\widetilde{L}(C))$ ).

Построим диффеоморфизм $g$ множества $V_{*}$ на $V_{*}^{\prime}$ такой, что:

1) $g$ оставляет инвариантным множество $\partial N_{\Omega}$ и имеет на каждой компоненте $l$ края в точности $r_{C(l)}$ седловых и $r_{C(l)}$ источниковых периодических точек;

2) для каждой точки $x \in \bigcup_{C \in \widehat{C}} f^{-1}(K(C))$ найдется номер $n$ такой, что $g^{n}(x) \in \bigcup_{C \in \widehat{C}} K(C)$

3) $\left.g\right|_{\cup_{C \in \widehat{C}}} \overline{K(C)}=\left.f\right|_{\cup_{C \in \widehat{C}}} \overline{K(C)}$.

Определим диффеоморфизм $f_{\Omega}$ подмногообразия $N_{\Omega}$ на себя по правилу

$$
f_{\Omega}(z)= \begin{cases}g(z), & z \in V_{*} \\ f(z), & z \in V\end{cases}
$$


По построению $\left.f_{\Omega}\right|_{V}=\left.f\right|_{V}$, множество $N_{\Omega} \backslash\left(\Omega \cup \partial N_{\Omega}\right)$ состоит из блуждаюших точек диффеоморфизма $f_{\Omega}$ и является объединением $m R(\Omega)$ непересекающихся между собой областей, гомеоморфных кольцу. Достижимая изнутри граница каждой такой области состоит в точности из одной связки $C$ множества $\Omega$ и одной компоненты края $\partial N_{\Omega}$ многообразия $N_{\Omega}$, содержащей в точности $r_{C}$ седловых и $r_{C}$ источниковых периодических точек диффеоморфизма $f_{\Omega}$ (рис. 6).

Построение диффеоморфизма $g$ множества $V_{*}$ на $V_{*}^{\prime}$ можно осушествить таким образом, что диффеоморфизм $f_{\Omega}$ обладает следующими свойствами:

а) для каждой граничной периодической точки $p \in \Omega$ диффеоморфизма $f_{\Omega}$ множество $\partial W^{s}(p)$ состоит в точности из одной источниковой периодической точки, принадлежащей $\partial N_{\Omega} ;$

б) неустойчивое многообразие каждой седловой периодической точки, принадлежащей $\partial N_{\Omega}$, трансверсально пересекается с устойчивыми многообразиями точек из $\Omega$ (рис. 6 ).

Покажем, что эйлерова характеристика $\chi\left(N_{i}\right)=2-2 q-R(\Omega)$ подмногообразия $N_{i}$ отрицательна. Если род $q$ подмногообразия $N_{i}$ больше нуля, то это очевидно. Предположим, что $q=0$. Рассмотрим диффеоморфизм $f_{\Omega}^{m}$, для которого подмногообразие $N_{i}$ является инвариантным. Тогда существует замкнутое подмногообразие $\widetilde{N}_{i}$, гомеоморфное двумерной сфере, и $A$-диффеоморфизм $f_{i}$ многообразия $\widetilde{N}_{i}$ такие, что:

1) $N_{i} \subset \tilde{N}_{i}$

2) неблуждающее множество диффеоморфизма $f_{i}$ состоит из одномерного аттрактора $\Omega_{i}$ и конечного числа периодических источниковых точек;

3) ограничение дифффеоморфизмов $f_{i}$ и $f^{m}$ на множестве $\Omega_{i}$ совпадают.

В силу [33] число связок любого аттрактора диффеоморфизма, заданного на многообразии нулевого рода, не менее четырех. То есть если многообразие $N_{i}$ имеет нулевой род, то оно имеет в этом случае $R(\Omega) \geqslant 4$ компонент края, и следовательно, эйлерова характеристика $\chi\left(N_{i}\right)=2-R(\Omega)$ отрицательна. Таким образом, эйлерова характеристика $\chi\left(N_{i}\right)=2-2 q-R(\Omega)$ подмногообразия $N_{i} \subset \widetilde{N}_{i}$ отрицательна в случае любого его рода $q$. Лемма 4 полностью доказана.

\section{§ 3. Инвариантность гетероклинических подстановок относительно топологической сопряженности}

В этом параграфе мы докажем леммы 6 и 7.

ДоКАЗАТЕЛЬСТво ЛЕммы 6 . Так как точки $x, y$ принадлежат одной траектории, то сушествует $k \in \mathbb{Z}$ такое, что $y=f^{k}(x)$. Тогда $y \in L^{s}\left(f^{k}(p)\right) \cap L^{u}\left(f^{k}(q)\right)$, где

$$
L^{s}\left(f^{k}(p)\right)=f^{k}\left(L^{s}(p)\right), \quad L^{u}\left(f^{k}(q)\right)=f^{k}\left(L^{u}(q)\right)
$$

- компоненты связности множеств

$$
W^{s}\left(f^{k}(p)\right) \backslash f^{k}(p), \quad W^{u}\left(f^{k}(q)\right) \backslash f^{k}(q),
$$

соответственно, содержащие точку $y$. 
Пусть $m \in \mathbb{Z}^{+}$наименьшее число, для которого

$$
f^{m}\left(L^{s}(p)\right)=L^{s}(p), \quad f^{m}\left(L^{u}(q)\right)=L^{u}(q) .
$$

Тогда

$$
\begin{aligned}
& f^{m}\left(L^{s}\left(f^{k}(p)\right)\right)=f^{m}\left(f^{k}\left(L^{s}(p)\right)\right)=f^{k}\left(f^{m}\left(L^{s}(p)\right)\right)=f^{k}\left(L^{s}(p)\right)=L^{s}\left(f^{k}(p)\right), \\
& f^{m}\left(L^{u}\left(f^{k}(q)\right)\right)=f^{m}\left(f^{k}\left(L^{u}(q)\right)\right)=f^{k}\left(f^{m}\left(L^{u}(q)\right)\right)=f^{k}\left(L^{u}(q)\right)=L^{u}\left(f^{k}(q)\right) .
\end{aligned}
$$

Откуда следует, что число $m$ является минимальным положительным числом, для которого компоненты $L^{s}\left(f^{k}(p)\right)$ и $L^{u}\left(f^{k}(q)\right)$ инвариантны относительно диффеоморфизма $f^{m}$. Так как диффеоморфизм $f^{k}$ отображает $L^{s}(p)$ на $L^{s}\left(f^{k}(p)\right), L^{u}(q)$ на $L^{u}\left(f^{k}(q)\right)$ с сохранением выбранных на них ориентаций (к точкам $p, f^{k}(p)$ и от точек $\left.q, f^{k}(q)\right)$ и

$$
\begin{aligned}
{\left[y, f^{m}(y)\right)^{s} \cap L^{u}\left(f^{k}(p)\right) } & =\left[f^{k}(x), f^{m}\left(f^{k}(x)\right)\right)^{s} \cap f^{k}\left(L^{u}(q)\right) \\
& =\left[f^{k}(x), f^{k}\left(f^{m}(x)\right)\right)^{s} \cap f^{k}\left(L^{u}(q)\right) \\
& =f^{k}\left(\left[x, f^{m}(x)\right)^{s} \cap L^{u}(q)\right),
\end{aligned}
$$

то $S(y)=S(x)$. Лемма 6 доказана.

ДоказАТЕЛЬСтво ЛЕммы 7. Пусть $G$ гетероклиническая траектория диффеоморфизма $f \in S(M)$ и $x \in L^{s}(p) \cap L^{u}(q)$ любая гетероклиническая точка, принадлежашая гетероклинической траектории $G$, где $p, q \in \Omega_{1}(f)$ и $L^{s}(p), L^{u}(q)$ есть компоненты связности множеств $W^{s}(p) \backslash p, W^{u}(q) \backslash q$, соответственно, содержащие точку $x$. Пусть $f^{\prime} \in S(M)$ диффеоморфизм, топологически сопряженный с диффеоморфизмом $f$, т.е. $f^{\prime}=g f g^{-1}$, где $g$ - гомеоморфизм многообразия $M$ на себя. Положим $x^{\prime}=g(x)$. Тогда точка $x^{\prime}$ принадлежит $L^{\prime s}\left(p^{\prime}\right) \cap L^{\prime u}\left(q^{\prime}\right)$ и является, следовательно, гетероклинической точкой диффеоморфизма $f^{\prime}$, где $p^{\prime}=g(p)$, $q^{\prime}=g(q)$ - периодические точки диффеоморфизма $f^{\prime}, L^{\prime s}\left(p^{\prime}\right)=g\left(L^{s}(p)\right), L^{\prime u}\left(q^{\prime}\right)=$ $g\left(L^{u}(q)\right)$ - компоненты связности множеств $W^{\prime s}\left(p^{\prime}\right) \backslash p^{\prime}, W^{\prime u}\left(q^{\prime}\right) \backslash q^{\prime}$, соответственно, содержащие точку $x^{\prime}$. Обозначим через $G^{\prime}=\bigcup_{n \in \mathbb{Z}} f^{\prime n}\left(x^{\prime}\right)$ гетероклиническую траекторию, содержащую точку $x^{\prime}$.

Так как гомеоморфизмы $f, f^{\prime}$ топологически сопряжены, то число $m$, являющееся наименьшим положительным числом, для которого компоненты $L^{s}(p), L^{u}(q)$ инвариантны, является одновременно наименшшим положительным числом, для которого инвариантны компоненты $L^{\prime s}\left(p^{\prime}\right), L^{\prime u}\left(q^{\prime}\right)$, и гомеоморфизм $g$ преобразует $L^{s}(p)$ на $L^{\prime s}\left(p^{\prime}\right), L^{u}(q)$ на $L^{\prime u}\left(q^{\prime}\right)$ с сохранением выбранных на них ориентаций (к точкам $p, p^{\prime}$ и от точек $q, q^{\prime}$, соответственно). Далее, имеем

$$
\begin{aligned}
{\left[x^{\prime}, f^{\prime m}\left(x^{\prime}\right)\right)^{s} \cap L^{\prime u}\left(p^{\prime}\right) } & =\left[g(x), f^{\prime m}(g(x))\right)^{s} \cap L^{\prime u}(g(p)) \\
& =\left[g(x), g\left(f^{m}(x)\right)\right)^{s} \cap g\left(L^{u}(p)\right)=g\left(\left[x, f^{m}(x)\right)^{s} \cap L^{u}(p)\right) .
\end{aligned}
$$

Отсюда получаем, что $S(x)=S\left(x^{\prime}\right)$, и следовательно, $S(G)=S\left(G^{\prime}\right)$. Лемма 7 доказана. 


\section{§4. Асимптотическое поведение прообразов устойчивых и неустойчивых многообразий на накрытии канонического носителя. Инвариантность алгебраических представлений одномерных аттракторов и репеллеров}

В этом параграфе мы приведем без доказательства ряд утверждений 1-4 об асимптотическом поведении прообразов устойчивых и неустойчивых многообразий точек из одномерных базисных множеств на накрытии его канонического носителя. Впервые утверждения 1-4 были установлены автором работы для ориентируемых базисных множеств в работах [8], [9]. В [11] Р. В. Плыкиньм было отмечена их справедливость для просторно расположенных базисных множеств, а затем автором и Х.Х. Калаем была установлена их справедливость для произвольных базисных множеств [12], [13].

Используя утверждения 1-4, мы докажем в этом параграфе леммы 5 и 8.

Зафиксируем номер $i \in\{1, \ldots, m\}$. Так как эйлерова характеристика подмногообразия $N_{i}$ отрицательна, то на плоскости Лобачевского, в реализации Пуанкаре на внутренности круга $|z|<1$ комплексной $z$-плоскости, сушествуют дискретная группа движений $F$, изоморфная фундаментальной группе подмногообразия $N_{i}$, и подмножество $H_{F}$ такие, что фактормножество $H_{F} / F$ гомеоморфно подмногообразию $N_{i}$. Обозначим через $\pi_{i}$ естественную проекцию $H_{F} \rightarrow N_{i}$ и через $f_{i}$ ограничение диффеоморфизма $f_{\Omega}^{m}$ на подмногообразие $N_{i}$. Из теории Нильсена (см., например, [35]) следует, что каждый элемент $\gamma$ группы $F$ (отличный от тождественного) имеет в точности две различные неподвижные точки, которые лежат на единичной окружности. Будем называть такие точки рациональными. Замыкание множества рациональных точек обозначим через $E$. Точки, принадлежашие дополнению на $E$ к множеству рациональных точек, будем называть иррациональныцми точками множества $E$.

Обозначим через $\bar{\Omega}_{i}$ полньй прообраз при отображении $\pi_{i}$ множества $\Omega_{i}$ на накрывающей $H_{F}$. Для точки $\bar{x} \in \bar{\Omega}_{i}$ обозначим через $w^{\sigma}(\bar{x})$ прообраз многообразия $W^{\sigma}(x)$, проходящий через точку $\bar{x}$, где $x=\pi(\bar{x})$.

УТВЕРЖДЕНИЕ 1. Пусть точка $\bar{x}$ принадлежит $\bar{\Omega}_{i} u w^{\sigma}(\bar{x})$ прообраз многообразия $W^{\sigma}(x)$, проходящий через точку $\bar{x}$, где $x=\pi(\bar{x})$. Тогда

1) кривая $w^{u}(\bar{x})$ имеет в точности две различнье граничные точки $u_{1}, u_{2}$, которые принадлежат множеству $Е$ и являются иррациональными точками (рис. 7а);

2) если многообразие $W^{s}(x)$ не содержит граничной периодической точки множества $\Omega_{i}$, то кривая $w^{s}(\bar{x})$ имеет в точности две различные граничнье точки $s_{1}, s_{2}$, которье принадлежат $E$ и являются ирраииональными точками, при этом пересечение $w^{u}(\bar{x}) \cap w^{s}(\bar{x})$ состоит в точности из одной точки $\bar{x}($ рис. $7 \mathrm{a})$;

3) если многообразие $W^{s}(x)$ содержит граничную периодическую точку р, то прообраз $w_{\infty}^{s}(\bar{p})(p=\pi(\bar{p}))$ компоненты $W_{\infty}^{s}(p)$, содержащий точку $\bar{x}$, имеет в точности две граничные точки, одна из которых есть точка $\bar{p}$, а другая $s_{\infty}$ принадлежит $E$ и является иррачиональ- 
ной точкой, при әтом пересечение $w^{u}(\bar{x}) \cap w^{s}(\bar{p})$ состочт в точности из одной точки $\bar{x}$ (рис. 7б);

4) граничные точки кривой $w^{u}(\bar{x})$ не совпадают с граничными точками кривой $w^{s}(\bar{x})$ в случае 2) и с граничной точкой $s_{\infty}$ кривой $w_{\infty}^{s}(\bar{p})$ в cлучае 3$)$;

5) если $w^{u}(\bar{x})$ не содержит прообраза граничной периодической точки, то в $\bar{\Omega}_{i}$ не существует точки $\bar{z}$ такой, что $w^{u}(\bar{z}) \cap w^{u}(\bar{x})=\varnothing$, и кривые $w^{u}(\bar{z}), w^{u}(\bar{x})$ имеют хотя бъ одну общую граничную точку на $E$;

6) если $w^{u}(\bar{x})$ содержстт прообраз $\bar{p}$ граничной периодической точки $p$, то в $\bar{\Omega}_{i}$ найдутся точки $\overline{q_{1}}, \overline{q_{2}}\left(\bar{q}_{1} \neq \bar{q}_{2}\right)$, являющиеся прообразами граничных периодических точек $q_{1}, q_{2}$ из $\Omega$, такие, что $w^{u}\left(\overline{q_{1}}\right)$ имеет $u_{1}$ одной из своих граничных точек, а $w^{u}\left(\overline{q_{2}}\right)$ имеет й $_{2}$ одной из своих граничных точек, где $u_{1}, u_{2}$ - граничные точки кривой $w^{u}(\bar{x})($ рис. $7 \mathrm{~B})$;

7) не существует точки $\bar{y} \in \bar{\Omega}$ такой, что $w^{u}(\bar{x}) \cap w^{u}(\bar{y})=\varnothing, w^{u}\left(\overline{q_{1}}\right) \cap$ $w^{u}(\bar{y})=\varnothing\left(w^{u}\left(\overline{q_{2}}\right) \cap w^{u}(\bar{y})=\varnothing\right)$ и $w^{u}(\bar{y})$ имеет $u_{1}\left(u_{2}\right)$ одной из своих граничных точек;

8) если $w_{*}^{s}(\bar{x}), w_{*}^{s}(\bar{y})$ компоненты связности множсеств $w^{s}(\bar{x}) \backslash \bar{x}, w^{s}(\bar{y}) \backslash \bar{y}$ такие, что $w^{s}(\bar{x}) \cap w^{s}(\bar{y})=\varnothing$, и компоненты $W_{*}^{s}(\pi(\bar{x}))=\pi\left(w_{*}^{s}(\bar{x})\right)$, $W_{*}^{s}(\pi(\bar{y}))=\pi\left(w_{*}^{s}(\bar{y})\right)$ содержат мнохсества, плотные в $\Omega_{i}$, тогда кривые $w_{*}^{s}(\bar{x}), w_{*}^{s}(\bar{y})$ имеют различные граничные точки на $E$.
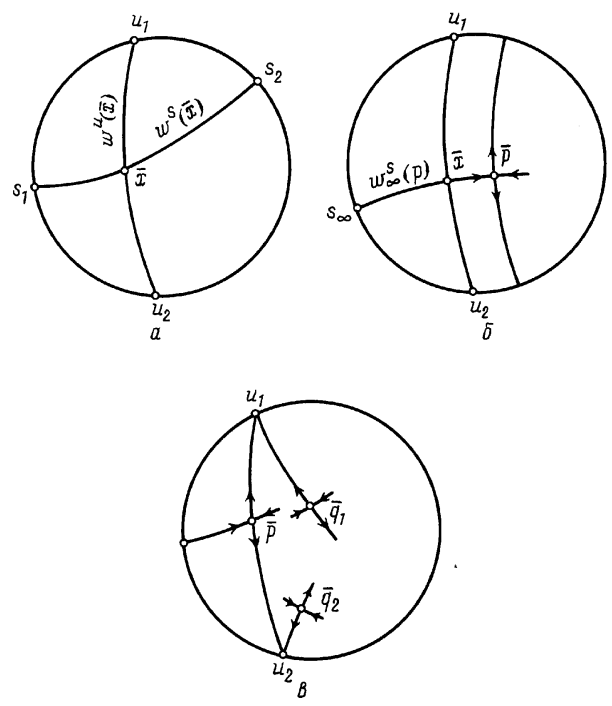

Рис. 7

Диффееоморфизм $f_{i}$ отображает подмногообразие $N_{i}$ на себя и индуцирует автоморфизм группы $F$, который определяется следующим образом. Обозначим через $\bar{f}_{i}$ отображение $H_{F}$ на себя, накрывающее отображение $f_{i}$, т.е. $\pi_{i} \bar{f}_{i}=f_{i} \pi_{i}$. Ото- 
бражение $\bar{f}_{i}$ индуцирует автоморфизм $\bar{f}_{i *}$ группы $F$, который задается формулой $\bar{f}_{i *}(\gamma)=\bar{f}_{i} \gamma \bar{f}_{i}^{-1}, \gamma \in F$.

В силу [35] любой автоморфизм $\tau$ групшы $F$ индуцирует единственным образом гомеоморфизм $\tau^{*}$ множества $E$ на себя. Обозначим через $\bar{f}_{i}^{*}$ гомеоморфизм множества $E$, индуцированный автоморфизмом $\bar{f}_{i *}$. Тогда гомеоморфизм $\bar{f}_{i}$ единственным образом продолжается на множество $E$ и совпадает на $E$ с гомеоморфизмом $\bar{f}_{i}^{*}$.

УТВЕРЖДЕНИЕ 2. Пусть р внутренняя периодическая точка диффеоморфизма $f_{i}$ периода $k$ и $\bar{f}_{i_{k}}$ отображение множества $H_{F}$ на себя, накрывающее отображение $f_{i}^{k}$, для которого некоторый прообраз $\bar{p}$ точки р является неподвижной точкой. Тогда либо гомеоморфизм $\bar{f}_{i_{k}}^{*}$, либо гомеоморфизм $\bar{f}_{i_{k}}^{* 2}$ имеет на Е в точности четыре неподвижные точки: притягивающие $u_{1}, u_{2}$ и отталкивающие $s_{1}, s_{2}$, где $u_{1}, u_{2}$ являются граничными точками кривой $w^{u}(\bar{p})$, a $s_{1}, s_{2}$ являются граничными точками кривой $w^{s}(\bar{p})$.

Справедливо и обратное утверждение.

УТВЕРЖДЕНИЕ 3. Пусть отображсение $\bar{f}_{i_{k}}$ является накрывающим для отображения $f_{i}^{k}$, и гомеоморфизм $\bar{f}_{i_{k}}^{*}$ имеет на множестве $Е$ в точности четыре неподвижные точки, которые являются иррациональными точками множества Е. Тогда диффеоморфизм $\bar{f}_{i_{k}}$ имеет неподвижную точку $\bar{p}$, образ $p=\pi(\bar{p})$ которой является внутренней периодической точкой диффеоморфизма $f_{i}$.

ДокАЗАТЕЛЬСтво ЛЕммы 5. Пусть $f^{\prime} \in S(M)$ диффеоморфизм, топологически сопряженный с диффеоморфизмом $f$, т.е. $f^{\prime}=g f g^{-1}$, где $g$-гомеоморфизм многообразия $M$ на себя, и $\Omega, \Omega^{\prime}$ одномерные базисные множества диффеоморфизмов $f, f^{\prime}$, соответственно, такие, что $\Omega^{\prime}=g(\Omega)$.

Пусть $m$ число $C$-плотных компонент в базисных множествах $\Omega, \Omega^{\prime}$ (число $m$ есть инвариант топологической сопряженности). Тогда можно построить канонические диффеоморфизмы $f_{\Omega}, f_{\Omega^{\prime}}^{\prime}$ и гомеоморфизм $\widetilde{g}: N_{\Omega} \rightarrow N_{\Omega^{\prime}}^{\prime}$ такие, что $f_{\Omega^{\prime}}^{\prime}=\widetilde{g} f_{\Omega} \widetilde{g}^{-1}$, и следовательно, $f_{\Omega^{\prime}}^{\prime m}=\widetilde{g} f_{\Omega}^{m} \widetilde{g}^{-1}$. Тогда существуют такие нумерации $C$-плотных компонент базисных множеств $\Omega, \Omega^{\prime}$, подмногообразия $N_{i} \subset N_{\Omega}$, $N_{i}^{\prime} \subset N_{\Omega^{\prime}},\left(\Omega_{i} \subset N_{\Omega}, \Omega_{i}^{\prime} \subset N_{\Omega^{\prime}}^{\prime}\right)$, диффеоморфизмы $f_{i}, f_{i}^{\prime}$, преобразуюшие $N_{i}, N_{i}^{\prime}$ на себя $\left(f_{i}, f_{i}^{\prime}\right.$ являются ограничениями диффеоморфизмов $f_{\Omega}^{m}, f_{\Omega^{\prime}}^{\prime m}$ на подмногообразия $N_{i}, N_{i}^{\prime}$ ), такие, что $f_{i}^{\prime}=g_{i} f_{i} g_{i}^{-1}$, где $g_{i}$ есть ограничение гомеоморфизма $\widetilde{g}$ на $N_{i}$.

Представим подмногообразие $N_{i}\left(N_{i}^{\prime}\right)$ в виде $H_{F} / F\left(H_{F^{\prime}}^{\prime} / F^{\prime}\right)$ с естественной проекцией $\pi_{i}\left(\pi_{i}^{\prime}\right)$, где $H_{F}\left(H_{F^{\prime}}^{\prime}\right)$ подмножество на плоскости Лобачевского и $F\left(F^{\prime}\right)$ - дискретная группа движений, изоморфная фундаментальной группе многообразия $N_{i}\left(N_{i}^{\prime}\right)$. Тогда сушествуют отображение $\bar{f}_{i}\left(\bar{f}_{i}^{\prime}\right)$ подмножества $H_{F}\left(H_{F^{\prime}}^{\prime}\right)$ на себя, накрывающее отображение $f_{i}\left(f_{i}^{\prime}\right), \pi_{i} \bar{f}_{i}=f_{i} \pi_{i}\left(\pi_{i}^{\prime} \bar{f}_{i}^{\prime}=f_{i}^{\prime} \pi_{i}^{\prime}\right)$, отображение $\bar{g}_{i}$ подмножества $H_{F}$ на $H_{F^{\prime}}^{\prime}, \pi_{i}^{\prime} \bar{g}_{i}=g_{i} \pi_{i}$, такие, что $\bar{f}_{i}^{\prime}=\bar{g}_{i} \bar{f}_{i} \bar{g}_{i}^{-1}$. 
Зададим автоморфизмы группы $F, F^{\prime}$ формулами:

$$
\begin{aligned}
\tau_{i}(\gamma)=\bar{f}_{i *}(\gamma) & =\bar{f}_{i} \gamma \bar{f}_{i}^{-1}, & & \gamma \in F, \\
\tau_{i}^{\prime}\left(\gamma^{\prime}\right)=\bar{f}_{i *}^{\prime}\left(\gamma^{\prime}\right) & =\bar{f}_{i}^{\prime} \gamma^{\prime} \bar{f}_{i}^{-1}, & & \gamma^{\prime} \in F^{\prime}
\end{aligned}
$$

Зададим автоморфизмы групा $F, F^{\prime}$ формулами:

$$
\begin{gathered}
\tau_{i}(\gamma)=\bar{f}_{i *}(\gamma)=\bar{f}_{i} \gamma \bar{f}_{i}^{-1}, \gamma \in F \\
\tau_{i}^{\prime}\left(\gamma^{\prime}\right)=\bar{f}_{i *}^{\prime}\left(\gamma^{\prime}\right)=\bar{f}_{i}^{\prime} \gamma^{\prime} \bar{f}_{i}^{-1}, \gamma^{\prime} \in F^{\prime} .
\end{gathered}
$$

Зададим изоморфизм $\psi$ группы $F$ на группу $F^{\prime}$ формулой:

$$
\psi(\gamma)=\bar{g}_{i *}(\gamma)=\bar{g}_{i} \gamma \bar{g}_{i}^{-1}, \quad \gamma \in F
$$

Тогда для любой точки $\bar{x} \in H_{F^{\prime}}^{\prime}$ имеем

$$
\begin{aligned}
\bar{f}_{i}^{\prime}(\gamma(x)) & =\bar{g}_{i}\left(\bar{f}_{i}\left(\bar{g}_{i}^{-1}(\gamma(x))\right)\right)=\bar{g}_{i}\left(\bar{f}_{i}\left(\psi^{-1}(\gamma)\left(\bar{g}_{i}^{-1}(x)\right)\right)\right) \\
& =\bar{g}_{i}\left(\tau_{i} \psi^{-1}(\gamma)\left(\bar{f}_{i}\left(\bar{g}_{i}^{-1}(x)\right)\right)\right)=\psi \tau_{i} \psi^{-1}(\gamma)\left(\bar{g}_{i}\left(\bar{f}_{i}\left(\bar{g}_{i}^{-1}(x)\right)\right)\right) \\
& =\psi \tau_{i} \psi^{-1}(\gamma)\left(\bar{f}_{i}^{\prime}(x)\right) .
\end{aligned}
$$

Отсюда получаем $\tau_{i}^{\prime}=\psi \tau_{i} \psi^{-1}$, т.е. автоморфизмы $\tau_{i}$ и $\tau_{i}^{\prime}$ алгебраически сопряжены. Лемма 5 доказана.

Предположим теперь, что алгебраические представления $\left(F, \tau_{i}\right)_{\Omega_{i}},\left(F^{\prime}, \tau_{i}^{\prime}\right)_{\Omega_{i}^{\prime}}$ алгебраически сопряжены, т.е. существуют такие накрывающие отображения $\bar{f}_{i}$, $\bar{f}_{i}^{\prime}$, и изоморфизм $\psi$ группы $F$ на группу $F^{\prime}$ такой, что $\bar{f}_{i *}^{\prime}=\psi \bar{f}_{i *} \psi^{-1}$. В силу [35] автоморфизм $\psi$ индуцирует единственньм образом гомеоморфизм $\psi^{*}$ множества $E$ на множество $E^{\prime}$, удовлетворяющий условию $\psi^{*}(\gamma(e))=\psi(\gamma)\left(\psi^{*}(e)\right), e \in E$. Поэтому получаем $\bar{f}_{i}^{\prime *}=\psi^{*} \bar{f}_{i}^{*} \psi^{*-1}$.

Следующееутверждение 4 являетсянепринципиальноймодификацией леммы 4.1 из [9] (аналогичные утверждения использовались в близкой к нам ситуации в [13]).

УТВЕРЖДЕНИЕ 4. 1) Если точка $\bar{p} \in \bar{\Omega}_{i}$ является прообразом внутренней периодической точки $p \in \Omega_{i}$, то найдется единственная точка $\bar{p}^{\prime} \in \bar{\Omega}_{i}^{\prime}$, являющаяся прообразом внутренней периодической точки $p^{\prime} \in \Omega_{i}^{\prime}$ того же периода, что и точка р, такая, что образы при отображении $\psi^{*}$ граничных точек кривых $w^{u}(\bar{p}), w^{s}(\bar{p})$ являются граничными точками кривых $w^{\prime u}\left(\bar{p}^{\prime}\right)$, $w^{\prime s}\left(\bar{p}^{\prime}\right)$, соответственно.

2) Если точка $\bar{p} \in \bar{\Omega}_{i}$ является прообразом граничной периодической точки $p \in \Omega_{i}$, то найдется единственная точка $\bar{p}^{\prime} \in \bar{\Omega}_{i}^{\prime}$, являющаяся прообразом граничной периодической точки $p^{\prime} \in \Omega_{i}^{\prime}$ того же периода, что и точка $p$, такая, что образы при отображсении $\psi^{*}$ граничных точек кривой $w^{u}(\bar{p})$ и граничной точки кривой $w_{\infty}^{s}(\bar{p})$, принадлежащей множеству Е, являются граничнбци точками кривой $w^{\prime u}\left(\bar{p}^{\prime}\right)$ и кривой $w_{\infty}^{\prime s}\left(\bar{p}^{\prime}\right)$, соответственно. 
3) Если точка $\bar{x} \in \bar{\Omega}_{i}$ такова, что кривая $w^{s}(\bar{x})$ не содержит прообраз граничной периодической точки множсества $\Omega$, то найдется точка $\bar{x}^{\prime} \in \bar{\Omega}_{i}^{\prime}$ такая, что образы при отобрахсении $\psi^{*}$ граничных точек кривых $w^{u}(\bar{x}), w^{s}(\bar{x})$ являются граничными точками кривых $w^{\prime u}\left(\bar{x}^{\prime}\right), w^{\prime s}\left(\bar{x}^{\prime}\right)$, соответственно.

Обозначим через $\pi\left(\pi^{\prime}\right)$ взаимно однозначное отображение множества вершин и ребер графа $G(f)\left(G^{\prime}\left(f^{\prime}\right)\right)$ на множество периодических точек, $C$-плотных компонент одномерных базисных множеств, гетероклинических компонент и компонент связности из множеств $W_{P_{1}}$ и $W_{\Gamma}\left(W_{P_{1}^{\prime}}^{\prime}\right.$ и $\left.W_{\Gamma^{\prime}}^{\prime}\right)$ диффеоморфизма $f\left(f^{\prime}\right)$.

ДокАЗАТЕЛьство лЕммы 8. Пусть $b$ вершина графа $G(f)$, принадлежашая множеству $B\left(w_{i}\right)$, и $p=\pi(b)$ соответствуюшая ей граничная периодическая точка множества $\Omega_{i}$. Рассмотрим любой прообраз $\bar{p}$ точки $p$ на накрытии $H_{F}$ и обозначим через $\bar{p}^{\prime}$ соответствующую ей точку, определяемую пунктом 2) утверждения 4 . Положим $p^{\prime}=\pi_{i}\left(\bar{p}^{\prime}\right)$ (по построению $p^{\prime} \in \Omega_{i}^{\prime}$ ) и обозначим через $b^{\prime}$ точку на графе $G^{\prime}\left(f^{\prime}\right)$, соответствующую граничной периодической точке $p^{\prime}\left(p^{\prime}=\pi^{\prime}\left(b^{\prime}\right)\right)$. Тогда $b^{\prime} \in B^{\prime}\left(w_{i}^{\prime}\right)$ и мы положим $\psi_{*}(b)=b^{\prime}$.

Пусть $l$ ребро графа $G(f)$, принадлежащее множеству $R\left(w_{i}\right)$. Тогда существует граничная периодическая точка $q$, принадлежащая $C$-плотной компоненте $\Omega_{i}$, такая, что одна из компонент связности множества $W^{u}(q) \backslash q$, обозначим ее через $L_{*}^{u}(q)$, соответствует ребру $l\left(\pi(l)=L_{*}^{u}(q)\right)$. Рассмотрим любой прообраз $\bar{q} \in \bar{\Omega}$ точки $q$ на накрытии $H_{F}$ и обозначим через $l_{*}^{u}(\bar{q})$ прообраз компоненты $L_{*}^{u}(q)$, для которого точка $\bar{q}$ является граничной точкой на $H_{F}$. Обозначим через $u_{1}$ граничную точку кривой $l_{*}^{u}(\bar{q})$ и через $s_{\infty}$ граничную точку кривой $w_{\infty}^{s}(\bar{q})$, принадлежащие множеству $E$. Обозначим через $u_{1}^{\prime}, s_{\infty}^{\prime}$ образы точек $u_{1}, s_{\infty}$ при отображении $\psi^{*}$. Тогда в силу утверждения 4 существует единственная точка $\bar{q}^{\prime} \in \overline{\Omega_{i}^{\prime}}$, являющаяся прообразом граничной периодической точки $q^{\prime} \in \Omega_{i}^{\prime}$ того же периода, что и точка $q$, такая, что точка $u_{1}^{\prime}$ является граничной точкой кривой $l^{\prime u}\left(\bar{q}^{\prime}\right)$, являющейся одной из компонент связности множества $w^{\prime u}(\bar{q}) \backslash \bar{q}$, а точка $s_{\infty}^{\prime}$ является граничной точкой компоненты $w_{\infty}^{\prime s}(\bar{q})$. Положим $L^{\prime u}\left(\bar{p}^{\prime}\right)=\pi_{i}^{\prime}\left(l^{\prime u}\left(\bar{q}^{\prime}\right)\right)$ и обозначим через $l^{\prime}$ ребро графа $G^{\prime}\left(f^{\prime}\right)$, соответствуюшее компоненте $L^{\prime u}\left(\bar{p}^{\prime}\right)\left(L^{\prime u}\left(\bar{p}^{\prime}\right)=\pi^{\prime}\left(l^{\prime}\right)\right)$. Тогда $\psi_{*}(l)=l^{\prime}$.

Таким образом, получаем взаимно однозначное отображение $\psi_{*}$ множества $B\left(w_{i}\right) \cup R\left(w_{i}\right)$ на множество $B^{\prime}\left(w_{i}^{\prime}\right) \cup R^{\prime}\left(w_{i}^{\prime}\right)$. Лемма 8 доказана.

\section{§5. Доказательство необходимых и достаточных условий топологической сопряженности}

Этот параграф посвящен доказательству теоремы 2. Доказательство необходимости условий теоремы 2 приводится в лемме 9. Доказательство достаточности условий теоремы состоит в построении сопрягающего гомеоморфизма, который последовательно строится в леммах 10-12.

ДокАЗАТЕЛЬСтво ЛЕммы 9. Пусть $f^{\prime} \in S(M)$ диффеоморфизм, топологически сопряженный с диффеоморфизмом $f$, т.е. $f^{\prime}=g f g^{-1}$, где $g$-гомеоморфизм многообразия $M$ на себя, и $G^{\prime}\left(f^{\prime}\right)$ граф, соответствующий диффеоморфизму $f^{\prime}$. Определим изоморфизм $\eta$ графов $G^{*}(f)$ и $G^{\prime *}\left(f^{\prime}\right)$ следующим образом. Пусть $e$ 
вершина или ребро графа $G(f)$, положим $\eta(e)=\pi^{\prime-1}(g(\pi(e)))$. Покажем, что изоморфизм $\eta$ удовлетворяет всем требованиям изоморфизма различающих графов $G^{*}(f)$ и $G^{*}\left(f^{\prime}\right)$ в определении 10. Условия 1$\left.)-4\right)$ определения 10 следуют непосредственно из топологической сопряженности диффеоморфизмов $f, f^{\prime}$ посредством гомеоморфизма $g$. Условие 5) выполняется в силу леммы 5 , определения изоморфизма $\eta$ и определения отображения $\psi_{*}$ в лемме 8 . Если $b, b^{\prime}$ вершины графов $G(f), G^{\prime}\left(f^{\prime}\right)$, соответствующие периодическим точкам из тривиальных базисных множеств, $b^{\prime}=\eta(b)$ и гомеоморфизм $g$ сохраняет ориентацию многообразия $M$, то снабдим множества $U(b), U^{\prime}\left(b^{\prime}\right)$ одинаково ориентированными нумерациями (например, положительно ориентированными нумерациями) таким образом, что номера изоморфных при $\eta$ ребер совпадают. Если гомеоморфизм $g$ меняет ориентацию $M$, то снабдим множества $U(b), U^{\prime}\left(b^{\prime}\right)$ противоположно ориентированными ориентациями (например, в $U(b)$ положительно ориентированной нумерацией, а в $U^{\prime}\left(b^{\prime}\right)$ отрицательно ориентированной нумерацией) таким образом, что номера изоморфных при $\eta$ ребер совпадают. Тогда для изоморфизма $\eta$ вьполняется условие 6) из определения 10.

Обозначим через $r\left(r^{\prime}\right)$ минимальное положительное число, для которого все точки из тривиальных базисных множеств диффеоморфизма $f\left(f^{\prime}\right)$ неподвижны относительно $f^{r}\left(f^{\prime r^{\prime}}\right)$ и компоненты связности множества $W^{\sigma}(p) \backslash p\left(W^{\prime \sigma}\left(p^{\prime}\right) \backslash p^{\prime}\right)$ инвариантны относительно $f^{r}\left(f^{\prime r^{\prime}}\right)$ для любой точки $p \in P_{1}\left(p^{\prime} \in P_{1}^{\prime}\left(f^{\prime}\right)\right)$. Так как $g$ есть топологическая сопряженность, то $r=r^{\prime}$. Пусть $\gamma, \gamma^{\prime}$ вершины графов $G(f), G^{\prime}\left(f^{\prime}\right)$, соответствуюшие гетероклиническим областям диффеоморфизмов $f, f^{\prime}, \gamma^{\prime}=\eta(\gamma)$ и $l^{\prime \sigma}=\eta\left(l^{\sigma}\right), l^{\sigma} \in Q^{\sigma}(\gamma), l^{\prime \sigma} \in Q^{\prime \sigma}\left(\gamma^{\prime}\right)$.

Пусть $L^{\sigma}=\pi\left(l^{\sigma}\right)$ и $x_{1}^{\sigma}, \ldots, x_{N\left(l^{\sigma}\right)}^{\sigma}$ гетероклинические точки на кривой $L^{\sigma}$, занумерованные в соответствии с ориентацией на ней, выбранные таким образом, что на дуге $\left[x_{1}^{\sigma}, x_{N\left(l^{\sigma}\right)}^{\sigma}\right]^{\sigma} \subset L^{\sigma}$ нет гетероклинических точек, отличных от них, и принадлежащие по одной всем различным гетероклиническим траекториям диф्ффеоморфизма $f^{r}$, имеющим с $L^{\sigma}$ не пустое пересечение.

Определим отображение $\varphi_{\gamma}^{l^{\sigma}}$ из множества $\left\{1, \ldots, N\left(l^{\sigma}\right)\right\}$ в множество $Q^{\bar{\sigma}}(\gamma)$, полагая $\varphi_{\underline{\gamma}}^{l^{\sigma}}(j)=l^{\bar{\sigma}}$, где $l^{\bar{\sigma}}$ является ребром, для которого $L^{\bar{\sigma}} \cap L^{\sigma}=x_{j}^{\sigma}$ $\left(L^{\bar{\sigma}}=\pi\left(l^{\bar{\sigma}}\right)\right)$. Пуусть $L^{\prime \sigma}=\pi^{\prime}\left(l^{\prime \sigma}\right)=g\left(L^{\sigma}\right)$. Положим $x_{j}^{\prime \sigma}=g\left(x_{j}^{\sigma}\right)$. Так как $g$ есть топологическая сопряженность, то точки $x_{1}^{\prime \sigma}, \ldots, x_{N\left(l^{\sigma}\right)}^{\prime \sigma}$ являются гетероклиническими точками диффеоморфизма $f^{\prime}$, принадлежат различным гетероклиническим траекториям и на дуге $\left[x_{1}^{\prime \sigma}, x_{N\left(l^{\sigma}\right)}^{\prime \sigma}\right]^{\sigma} \subset L^{\prime \sigma}$ нет гетероклинических точек диффеоморфизма $f^{\prime}$, отличных от них. Поэтому число $N\left(l^{\sigma}\right)$ различных гетероклинических траекторий диффеоморфизма $f$, имеющих непустое пересечение с компонентой $L^{\sigma}$, совпадает с числом $N^{\prime}\left(l^{\prime \sigma}\right)$ различных гетероклинических траекторий диффеоморфизма $f^{\prime}$, имеющих непустоепересечение с компонентой $L^{\prime \sigma}$.

Определим отображение $\varphi_{\gamma^{\prime}}^{\prime l^{\prime \sigma}}$ измножества $\left\{1, \ldots, N^{\prime}\left(l^{\prime \sigma}\right)\right\}$ в множество $Q^{\prime \bar{\sigma}}\left(\gamma^{\prime}\right)$, полагая $\varphi_{\gamma^{\prime}}^{\prime l^{\prime \sigma}}(j)=l^{/ \bar{\sigma}}$, где $l^{\prime \bar{\sigma}}$ является ребром, для которого $L^{\prime \bar{\sigma}} \cap L^{\prime \sigma}=x_{j}^{\prime}$. По построению изоморфизма $\eta$ имеем $l^{\prime \bar{\sigma}}=\eta\left(l^{\bar{\sigma}}\right)$.

Таким образом, мы можем оснастить графы $G^{*}(f), G^{*}\left(f^{\prime}\right)$ отображениями $\varphi_{\gamma}^{l^{\sigma}}$, $\varphi_{\gamma^{\prime}}^{\prime l^{\prime \sigma}}$ такими, что выполняется условие $\varphi_{\gamma^{\prime}}^{l^{\prime \sigma}}=\eta\left(\varphi_{\gamma}^{l^{\sigma}}\right)$.

Зададим отображения $\psi_{\gamma}^{l^{\sigma}}\left(\psi_{\gamma^{\prime}}^{\prime l^{\prime \sigma}}\right)$ из множества $\left\{1, \ldots, N\left(l^{\sigma}\right)\right\}\left(\left\{1, \ldots, N^{\prime}\left(l^{\prime \sigma}\right)\right\}\right)$ 
в множество подстановок, полагая $\psi_{\gamma}^{l^{\sigma}}(j)=S\left(x_{j}\right)\left(\psi_{\gamma^{\prime}}^{\prime l^{\prime \sigma}}(j)=S^{\prime}\left(x_{j}^{\prime}\right)\right)$. Тогда в силу леммы $7 S\left(x_{j}\right)=S^{\prime}\left(x_{j}^{\prime}\right)$, т.е. $\psi_{\gamma}^{l^{\sigma}}=\psi_{\gamma^{\prime}}^{\prime l^{\prime \sigma}}$. Таким образом, мы можем оснастить графы $G^{*}(f), G^{*}\left(f^{\prime}\right)$ отображениями $\varphi_{\gamma}^{l^{\sigma}}, \psi_{\gamma^{\prime}}^{\prime l^{\prime}}$ такими, что выполняется условие $\psi_{\gamma^{\prime}}^{\prime l^{\prime \sigma}}=\psi_{\gamma}^{l^{\sigma}}$.

Зададим отображения $\phi_{\gamma}^{l^{\sigma}}\left(\phi_{\gamma^{\prime}}^{\prime l^{\prime \sigma}}\right)$ из множества $\left\{1, \ldots, N\left(l^{\sigma}\right)\right\}\left(\left\{1, \ldots, N^{\prime}\left(l^{\prime \sigma}\right)\right\}\right)$ в множество $\{-1,1\}$, полагая $\phi_{\gamma}^{l^{\sigma}}(j)=J\left(x_{j}\right)\left(\phi_{\gamma^{\prime}}^{\prime l^{\prime}}(j)=J^{\prime}\left(x_{j}^{\prime}\right)\right)$. Тогда если гомеоморфизм $g$ сохраняет (меняет) ориентацию $M$, то $J^{\prime}\left(x_{j}^{\prime}\right)=J\left(x_{j}\right)\left(J^{\prime}\left(x_{j}^{\prime}\right)=-J\left(x_{j}\right)\right)$. В силу правила введения ориентированных нумераций в множествах $U(b), U^{\prime}\left(b^{\prime}\right)$, $b^{\prime}=\eta(b)$, имеем что $\phi_{\gamma^{\prime}}^{\prime l^{\prime}}=\nu \phi_{\gamma}^{l^{\sigma}}$, где $\nu=1(\nu=-1)$, если ориентации в нумерациях множеств $U(b), U^{\prime}\left(b^{\prime}\right)$ совпадают (противоположны). Таким образом, для изоморфизма $\eta$ выполняется условие 7) определения 10. Лемма 9 полностью доказана.

Далее будем предполагать, что выполнены достаточные условия теоремы 2, т.е. различаюшие графы $G^{*}(f), G^{*}(f)$ диффеоморфизмов $f, f^{\prime} \in S(M)$ изоморфны в силу изоморфизма $\eta$ такого, что $P^{\prime}\left(f^{\prime}\right)=\eta P(f) \eta^{-1}$.

Будем говорить, что некоторые объекты, связанные с диффеоморфизмами $f$ и $f^{\prime}$ (периодические точки, гетероклинические области, компоненты связности устойчивых и неустойчивых многообразий, одномерные аттракторы и репеллеры и т. п.), являются изоморфными, если они соответствуют изоморфным объектам в графах $G^{*}(f)$ и $G^{*}\left(f^{\prime}\right)$.

Пусть $\Omega, \Omega^{\prime}$, изоморфные одномерные базисные множества диффеоморфизмов $f$ и $f^{\prime}$, соответственно, и $M_{\Omega}, M_{\Omega^{\prime}}^{\prime}$ их канонические окрестности. Предположим для определенности, что множества $\Omega, \Omega^{\prime}$ являются аттракторами (если $\Omega, \Omega^{\prime}$ являются репеллерами, то все нижеприведенные утверждения переформулируются с очевидными изменениями).

Так как аттракторам $\Omega, \Omega^{\prime}$ соответствуют изоморфные множества в графах $G^{*}(f)$ и $G^{\prime *}\left(f^{\prime}\right)$ и $P^{\prime}\left(f^{\prime}\right)=\eta P(f) \eta^{-1}$, то аттракторы $\Omega, \Omega^{\prime}$ состоят из одинакового числа $m C$-плотных компонент и можно так выбрать их нумерации, что для каждой $C$-плотной компоненты $\Omega_{i} \subset \Omega$ найдется $C$-плотная компонента $\Omega_{i}^{\prime} \subset \Omega^{\prime}$ такая, что алгебраические представления $\left(F, \tau_{i}\right)_{\Omega_{i}},\left(F^{\prime}, \tau_{i}^{\prime}\right)_{\Omega_{i}^{\prime}}$ алгебраически сопряжены. Пусть $\left(N_{\Omega}, f_{\Omega}\right),\left(N_{\Omega^{\prime}}^{\prime}, f_{\Omega^{\prime}}^{\prime}\right)$ канонические формы аттракторов $\Omega, \Omega^{\prime}$. Зафиксируем $i \in\{1, \ldots, m\}$ и рассмотрим подмногообразия $N_{i}, N_{i}^{\prime}$, инвариантные относительно диффеоморифзмов $f_{\Omega}^{m}, f_{\Omega^{\prime}}^{\prime m}$. Представим $N_{i}, N_{i}^{\prime}$ как фактормножества $H_{F} / F$, $H_{F^{\prime}}^{\prime} / F^{\prime}$ и обозначим через $\pi_{i}, \pi_{i}^{\prime}$ естественные проекции $H_{F}, H_{F^{\prime}}^{\prime}$ на $N_{i}, N_{i}^{\prime}$, соответственно, где $H_{F}, H_{F^{\prime}}^{\prime}$ - подмножества плоскости Лобачевского и $F, F^{\prime}$ - дискретные группы движений. Обозначим через $f_{i}, f_{i}^{\prime}$ ограничения отображений $f_{\Omega}^{m}$, $f_{\Omega^{\prime}}^{\prime m}$ на подмногообразия $N_{i}, N_{i}^{\prime}$, соответственно.

Из определения алгебраической сопряженности представлений $\left(F, \tau_{i}\right)_{\Omega_{i}}$, $\left(F^{\prime}, \tau_{i}^{\prime}\right)_{\Omega_{i}^{\prime}}$ следует, что существуют накрьвающие отображения $\bar{f}_{i}, \bar{f}_{i}^{\prime}$ и алгебраический изоморфизм $\psi$ группы $F$ на группу $F^{\prime}$ такие, что $\bar{f}_{i *}^{\prime}=\psi \bar{f}_{i *} \psi^{-1}$. Изоморфизм $\psi$ индуцирует единственным образом гомеоморфизм $\psi^{*}$ множества $E$ на множество $E^{\prime}$, удовлетворяющий условию $\psi^{*}(\gamma(e))=\psi(\gamma)\left(\psi^{*}(e)\right)$. Поэтому получаем $\bar{f}_{i}^{\prime *}=\psi^{*} \bar{f}_{i}^{*} \psi^{*-1}$. 
Обозначим через $\bar{\Omega}_{i}\left(\bar{\Omega}_{i}^{\prime}\right)$ полный прообраз множества $\Omega_{i}\left(\Omega_{i}^{\prime}\right)$ при проекции $\pi_{i}\left(\pi_{i}^{\prime}\right)$.

Лемма 10. Пусть $\Omega, \Omega^{\prime}$ изоморфные одномерные аттракторы диффеоморфизмов $f$ и $f^{\prime}$, соответственно. Тогда существует гомеоморфизм $g$ множества $\Omega$ на множество $\Omega^{\prime}$ такой, что:

1) $\left.f^{\prime}\right|_{\Omega^{\prime}}=\left.g f g^{-1}\right|_{\Omega^{\prime}}$

2) если $x, y \in \Omega(x \neq y)$ такие, что $y \in W^{s}(x)$ u $(x, y)^{s} \cap \Omega=\varnothing$, тогда $g(y) \in W^{\prime s}(g(x)) u(g(x), g(y))^{s} \cap \Omega^{\prime}=\varnothing$.

ДокАЗАТЕЛЬСтво. Аналогично $[9, \S 6]$ зададим отображение $\bar{g}_{i}$ полного прообраза $\bar{\Omega}_{i}$ множества $\Omega_{i}$ на полный прообраз $\bar{\Omega}_{i}^{\prime}$ множества $\Omega_{i}^{\prime}$ следуюшим образом. Пусть $\bar{x}$ произвольная точка множества $\bar{\Omega}_{i}$. Тогда для нее могут быть в точности две возможности:

1) кривая $w^{s}(\bar{x})$ не содержит прообраза граничной периодической точки множества $\Omega$;

$2)$ кривая $w^{s}(\bar{x})$ содержит прообраз $\bar{p}$ граничной периодической точки $p$ множества $\Omega$.

В первом случае согласно утверждению 4 существует точка $\overline{x^{\prime}} \in \bar{\Omega}_{i}^{\prime}$ такая, что граничные точки кривых $w^{\prime u}\left(\overline{x^{\prime}}\right), w^{\prime s}\left(\overline{x^{\prime}}\right)$ являются образами при отображении $\psi^{*}$ граничньгх точек кривых $w^{u}(\bar{x}), w^{s}(\bar{x})$, соответственно.

Во втором случае рассмотрим кривую $w_{\infty}^{s}(\bar{p})$, содержашую кривую $w_{\infty}^{s}(\bar{x})$. Здесь $w_{\infty}^{s}(\bar{x})$ есть прообраз кривой $W_{\infty}^{s}(x)(x=\pi(\bar{x}))$, которая является компонентой связности множества $W^{s}(x) \backslash x$, принадлежашей компоненте $W_{\infty}^{s}(p)$. В силу утверждения 4 сушествует точка $\bar{p}^{\prime} \in \bar{\Omega}_{i}^{\prime}$, являюшаяся прообразом граничной периодической точки $p^{\prime}$ множества $\Omega^{\prime}$, такая, что граничная точка кривой $w_{\infty}^{\prime s}\left(\bar{p}^{\prime}\right)$, лежащая на $E^{\prime}$, является образом при отображении $\psi^{*}$ граничной точки кривой $w_{\infty}^{s}(\bar{p})$, лежащей на $E$. При этом граничные точки кривой $w^{\prime u}\left(\bar{p}^{\prime}\right)$ являются образом при $\psi^{*}$ граничных точек кривой $w^{u}(\bar{p})$. В силу утверждения 4 существует точка $\bar{y}^{\prime} \in \bar{\Omega}^{\prime}$ такая, что граничные точки кривой $w^{\prime u}\left(\bar{y}^{\prime}\right)$ являются образом при отображении $\psi^{*}$ граничных точек кривой $w^{u}(\bar{x})$. Так как отображение $\psi^{*}$ есть гомеоморфизм, то кривые $w^{\prime u}\left(\bar{p}^{\prime}\right) \cup \bar{p}^{\prime}$ и $w^{\prime u}\left(\bar{y}^{\prime}\right)$ непременно пересекаются в некоторой точке $\overline{x^{\prime}} \in \bar{\Omega}^{\prime}$. С учетом утверждения 4 получаем, что каждой точке $\bar{x} \in \bar{\Omega}_{i}$ ставится в соответствие единственная точка $\bar{x}^{\prime} \in \bar{\Omega}_{i}^{\prime}$. Обозначим полученное отображение через $\bar{g}_{i}$. Из конструкции отображения $\bar{g}_{i}$, близости устойчивых и неустойчивых многообразий точек из $\Omega_{i}$ и $\Omega_{i}^{\prime}$ на компактных множествах и свойств накрытий следует, что $\bar{g}_{i}$ есть гомеоморфизм множества $\bar{\Omega}_{i}$ на множество $\bar{\Omega}_{i}^{\prime}$. Так как гомеоморфизм $\psi^{*}$ удовлетворяет условиям $\psi^{*}(\gamma(e))=\psi(\gamma)\left(\psi^{*}(e)\right)$, $\bar{f}_{i}^{\prime *}=\psi^{*} \bar{f}_{i}^{*} \psi^{*-1}$, то из конструкции следует, что отображение $\bar{g}_{i}$ удовлетворяет условиям $\bar{g}_{i}(\gamma(\bar{x}))=\psi(\gamma)\left(\bar{g}_{i}(\bar{x})\right), \bar{f}_{i}^{\prime}\left(\bar{g}_{i}(x)\right)=\bar{g}_{i}\left(\bar{f}_{i}(x)\right)$. Но тогда сушествует гомеоморфизм $g_{i}$ множества $\Omega_{i}$ на множество $\Omega_{i}^{\prime}$, удовлетворяюший условию $\left.f_{i}^{\prime}\right|_{\Omega_{i}^{\prime}}=\left.g_{i} f_{i} g_{i}^{-1}\right|_{\Omega_{i}^{\prime}}$.

Определим гомеоморфизм $g$ множества $\Omega$ на множество $\Omega^{\prime}$ следующим образом. Для $x \in \Omega$ положим $g(x)=f^{\prime r}\left(g_{i}\left(f^{-r}(x)\right)\right)$, где $r \in\{0, \ldots, m-1\}$ такое, что $f^{-r}(x) \in \Omega_{i}$ (полагаем, что $f^{0}=\mathrm{id}$, где id - тождественное отображение). 
Непосредственно проверяется, что гомеоморфизм $g$ удовлетворяет условию $f_{\Omega^{\prime}}^{\prime}=g f g_{\Omega^{\prime}}^{-1}$. Так как по построению канонических форм аттракторов $\left.f_{\Omega}\right|_{\Omega}=\left.f\right|_{\Omega}$, $\left.f_{\Omega^{\prime}}^{\prime}\right|_{\Omega^{\prime}}=\left.f\right|_{\Omega^{\prime}}$, то $\left.f^{\prime}\right|_{\Omega^{\prime}}=\left.g f g^{-1}\right|_{\Omega^{\prime}}$.

Из утверждения 4 и конструкции гомеоморфизма $\bar{g}_{i}$ следует, что если $x, y \in \Omega$ $(x \neq y)$ такие, что $y \in W^{s}(x)$ и $(x, y)^{s} \cap \Omega=\varnothing$, тогда $g(y) \in W^{\prime s}(g(x))$ и $(g(x), g(y))^{s} \cap \Omega^{\prime}=\varnothing$. Лемма 10 полностью доказана.

Лемма 11. Пусть выполнены условия леммы 10. Тогда существует гомеоморфизм $h_{\Omega}$ множества $\bar{M}_{\Omega}$ намножсество $\bar{M}_{\Omega^{\prime}}^{\prime}$ такой, что $\left.h_{\Omega}\right|_{\Omega}=\left.g\right|_{\Omega}$ $\left.u f\right|_{M_{\Omega^{\prime}}^{\prime}}=\left.h_{\Omega} f h_{\Omega}^{-1}\right|_{M_{\Omega^{\prime}}^{\prime}} ^{\prime}$.

ДокАЗАТЕЛЬСтво. Зафиксируем $i \in\{1, \ldots, m\}$ и рассмотрим подмногообразия $M_{i}, M_{i}^{\prime}$, для которых вьполняются условия $\Omega_{i} \subset M_{i} \cup \Gamma_{i}, \Omega_{i}^{\prime} \subset M_{i}^{\prime} \cup \Gamma_{i}^{\prime}$. Тогда $f^{m}\left(\bar{M}_{i}\right)=\bar{M}_{i}, f^{\prime m}\left(\bar{M}_{i}^{\prime}\right)=\bar{M}_{i}^{\prime}$. Обозначим через $f_{i}, f_{i}^{\prime}$ ограничения отображений $f^{m}, f^{\prime m}$ на множества $\bar{M}_{i}, \bar{M}_{i}^{\prime}$, соответственно.

В силу леммы 10 сушествует гомеоморфизм $g_{i}$ множества $\Omega_{i}$ на множество $\Omega_{i}^{\prime}$ такой, что $\left.f_{i}^{\prime}\right|_{\Omega_{i}^{\prime}}=\left.g_{i} f_{i} g_{i}^{-1}\right|_{\Omega_{i}^{\prime}}$.

Пусть $p$ произвольная седловая периодическая точка, принадлежащая $\partial M_{i}$. В силу леммы 2 по крайней мере одна из компонент связности множества $W^{u}(p) \backslash p$ принадлежит $M_{i}$. Будем обозначать через $W_{*}^{u}(p)$ множество, состоящее из объединения компонент связности множества $W^{u}(p) \backslash p$, принадлежащих $M_{i}$.

Обозначим через $S_{i}, S_{i}^{\prime}$ множества всех седловых периодических точек, принадлежащих $\partial M_{i}, \partial M_{i}^{\prime}$, соответственно. Положим

$$
W_{* i}^{u}=\bigcup_{p \in S_{i}} W_{*}^{u}(p), \quad W_{* i}^{\prime u}=\bigcup_{p^{\prime} \in S_{i}^{\prime}} W_{*}^{\prime u}\left(p^{\prime}\right)
$$

Обозначим через $U_{i}, U_{i}^{\prime}$ множества всех источниковых периодических точек, принадлежаших $\partial M_{i}, \partial M_{i}^{\prime}$, соответственно.

Зададим отображение $h_{0}$ множества $S_{i} \cup U_{i}$ на множество $S_{i}^{\prime} \cup U_{i}^{\prime}$ следующим образом.

Если точка $x \in S_{i} \cup U_{i}$, то $x^{\prime}=h_{0}(x)=\pi^{\prime}\left(\eta\left(\pi^{-1}(x)\right)\right)$. Из условия 5) в определении 10 изоморфизма графов следует, что если $x \in \Gamma$, то $h_{0}(x)=g_{i}(x)$.

Зададим отображение $h_{1}$ множества $S_{i} \cup U_{i} \cup W_{* i}^{u}$ на множество $S_{i}^{\prime} \cup U_{i}^{\prime} \cup W_{* i}^{\prime u}$ следующим образом.

Если точка $x \in S_{i} \cup U_{i}$, то $h_{1}(x)=h_{0}(x)$.

Если точка $x \in W_{* i}^{u}$, то рассмотрим седловую периодическую точку $p \in S_{i}$ такую, что $x \in W_{* i}^{u}(p)$. Если $p$ является граничной периодической точкой множества $\Omega$, то положим $h_{1}(x)=g_{i}(x)$.

Если $p$ не является граничной периодической точкой, то из доказательства леммы 1 и конструкции множества $M_{i}$ в лемме 2 следует, что существуют граничные периодические точки $p_{1}, p_{2}\left(p_{1}\right.$ может совпадать с $\left.p_{2}\right)$ аттрактора $\Omega$ и компоненты связности $L_{1}^{u}, L_{2}^{u}$ множеств $W^{u}\left(p_{1}\right) \backslash p_{1}, W^{u}\left(p_{2}\right) \backslash p_{2}$, соответственно (если точки $p_{1}, p_{2}$ совпадают, то $L_{1}^{u}, L_{2}^{u}$ есть различные компоненты связности множества $\left.W^{u}\left(p_{1}\right) \backslash p_{1}\right)$, такие, что для любой точки $x \in L^{u}(p)$ найдутся точки $x_{1} \in L_{1}^{u}$, 
$x_{2} \in L_{2}^{u}$ такие, что $x_{2} \in W^{s}\left(x_{1}\right)$ и пересечение $W_{*}^{u}(p) \cap\left(x_{1}, x_{2}\right)^{s}$ состоит из единственной точки, являющейся точкой $x$.

Положим $p^{\prime}=h_{0}(p), p_{1}^{\prime}=g_{i}\left(p_{1}\right), p_{2}^{\prime}=g_{i}\left(p_{2}\right), x_{1}^{\prime}=g_{i}\left(x_{1}\right), x_{2}^{\prime}=g_{i}\left(x_{2}\right)$. В силу леммы $10 x_{2}^{\prime} \in W^{s}\left(x_{2}^{\prime}\right)$. Учитьвая выполнение условий 1$\left.)-4\right)$ определения 10 , получаем, что пересечение $W_{*}^{\prime}\left(p^{\prime}\right) \cap\left(x_{1}^{\prime}, x_{2}^{\prime}\right)^{s}$ непусто и состоит из единственной точки, которую обозначим через $x^{\prime}$. Положим $h_{1}(x)=x^{\prime}$. Из конструкции отображения $h_{1}$ и $C^{1}$-близости устойчивых и неустойчивых многообразий точек из $\Omega$ на компактных множествах следует, что $h_{1}$ является гомеоморфизмом.

Если $x^{\prime} \in S_{i}^{\prime} \cup U_{i}^{\prime}$, то

$$
\begin{aligned}
h_{1} f_{i} h_{1}^{-1}\left(x^{\prime}\right) & =h_{0} f_{i} h_{0}^{-1}\left(x^{\prime}\right)=\pi^{\prime} \eta \pi^{-1} \pi P^{m}(f) \pi^{-1} \pi \eta^{-1} \pi^{\prime-1}(x) \\
& =\pi^{\prime} \eta P^{m}(f) \eta^{-1} \pi^{\prime-1}(x)=\pi^{\prime} P^{\prime m}(f) \pi^{\prime-1}(x)=f^{\prime}\left(x^{\prime}\right) .
\end{aligned}
$$

Если $x^{\prime} \in W_{*}^{u}$, то

$$
\begin{aligned}
h_{1} f_{i} h_{1}^{-1}\left(x^{\prime}\right) & =h_{1} f_{i} h_{1}^{-1}\left(\left(x_{1}^{\prime}, x_{2}^{\prime}\right)^{s} \cap W_{*}^{\prime u}\left(p^{\prime}\right)\right) \\
& =h_{1} f_{i}\left(\left(g_{i}^{-1}\left(x_{1}^{\prime}\right), g_{i}^{-1}\left(x_{2}^{\prime}\right)\right)^{s} \cap W_{*}^{u}\left(h_{1}^{-1}\left(p^{\prime}\right)\right)\right) \\
& =h_{1}\left(\left(f_{i} g_{i}^{-1}\left(x_{1}^{\prime}\right), f_{i} g_{i}^{-1}\left(x_{2}^{\prime}\right)\right)^{s} \cap W_{*}^{u}\left(f_{i} h_{1}^{-1}\left(p^{\prime}\right)\right)\right) \\
& =\left(g_{i} f_{i} g_{i}^{-1}\left(x_{1}^{\prime}\right), g_{i} f_{i} g_{i}^{-1}\left(x_{2}^{\prime}\right)\right)^{s} \cap W_{*}^{u}\left(h_{1} f_{i} h_{1}^{-1}\left(p^{\prime}\right)\right) \\
& =\left(f_{i}^{\prime}\left(x_{1}^{\prime}\right), f_{i}^{\prime}\left(x_{2}^{\prime}\right)\right)^{s} \cap W^{u}\left(f_{i}^{\prime}\left(p^{\prime}\right)\right)=f_{i}\left(x^{\prime}\right) .
\end{aligned}
$$

Таким образом, гомеоморфизм $h_{1}$ преобразует множество $S_{i} \cup U_{i} \cup W_{* i}^{u}$ на множество $S_{i}^{\prime} \cup U_{i}^{\prime} \cup W_{* i}^{\prime u}$ и удовлетворяет условию

$$
\left.f_{i}^{\prime}\right|_{S_{i}^{\prime} \cup U_{i}^{\prime} \cup W_{* i}^{\prime u}}=\left.h_{1} f_{i} h_{1}^{-1}\right|_{S_{i}^{\prime} \cup U_{i}^{\prime} \cup W_{* i}^{\prime u}} .
$$

Целью дальнейших рассуждений является построение гомеоморфизма $H_{i}$ множества $\bar{M}_{i}$ на множество $\bar{M}_{i}^{\prime}$ такого, что

$$
\left.H_{i}\right|_{S_{i} \cup U_{i} \cup W_{* i}^{u}}=\left.h_{1}\right|_{S_{i} \cup U_{i} \cup W_{* i}^{u}}
$$

и $\left.f_{i}^{\prime}\right|_{\bar{M}_{i}^{\prime}}=\left.H_{i} f_{i} H_{i}^{-1}\right|_{\bar{M}_{i}^{\prime}}$.

Пусть $\alpha \in U_{i}$ источниковая периодическая точка периода $n_{0} \in \mathbb{Z}^{+}$. Обозначим через $W_{\alpha}^{s}$ объединение устойчивых многообразий $W^{s}(p)$ всех седловых периодических точек $p$, принадлежащих $S_{i}$, для которых выполняется условие $\alpha \in \overline{W^{s}(p)}$, и через $W_{\alpha \varnothing}^{s}$ объединение компонент связности $W_{\varnothing}^{s}(p)$ для всех граничных периодических точек $p$, принадлежащих $\Gamma_{i}$, для которых вьполняется условие $\alpha \in \overline{W_{\varnothing}^{s}(p)}$.

Пусть $L_{1}^{s}, \ldots, L_{k}^{s}$ все компоненты связности из множеств $W_{\alpha}^{s} \backslash S_{i}$ и $W_{\alpha \varnothing}^{s}$, для каждой из которых выполняется условие $\alpha \in \overline{L_{r}^{s}}, r=1, \ldots, k$, и занумерованные следуюшим образом. Выберем в окрестности точки $\alpha$ простую гладкую замкнутую кривую $L$ такую, что кривая $L$ ограничивает диск $D$, содержаший точку $\alpha$, 
и пересекается в точности в одной точке с каждой компонентой связности из множества $W_{\alpha}^{s} \backslash S_{i}$ и $W_{\alpha \varnothing}^{s}$, имеющей точку $\alpha$ в своем замыкании. Зададим в некоторой точке кривой $L$ репер $(\tau, n)$, согласованный с ориентацией многообразия $M$, такой, что вектор $\tau$ касается кривой $L$ и вектор $n$ направлен внутрь диска $D$. Зафиксируем направление обхода по кривой $L$, индуцированное вектором $\tau$. Занумеруем компоненты $L_{1}^{s}, \ldots, L_{k}^{s}$ в порядке, в котором они встречаются при обходе вдоль $L$, начиная с некоторой точки на $L$. Пусть $p_{1}, \ldots, p_{k}$ периодические точки из $S_{i} \cup \Gamma_{i}$ такие, что $L_{r}^{s} \in W^{s}\left(p_{r}\right)$ (если $p_{i}=p_{j}=p$ для некоторых $i, j \in\{1, \ldots, k\}$, то $L_{i}^{s}, L_{j}^{s}$ являются различными компонентами связности множеств $\left.W^{s}(p) \backslash p\right)$.

Выберем на каждой кривой $L_{r}^{s}$ точку $z_{r}$ таким образом, что если $f^{n_{0}}\left(z_{r}\right) \in L_{j}^{s}$, то $f^{n_{0}}\left(z_{r}\right) \in\left(z_{j}, p_{j}\right)^{s} \subset W^{s}\left(p_{j}\right), r, j \in\{1, \ldots, k\}\left(j=r\right.$, если $\left.f^{n_{0}}\left(L_{r}^{s}\right)=L_{r}^{s}\right)$.

По построению границы множества $M_{i}$ в лемме 2 точка $\alpha$ лежит в замыкании последовательности кривых $L_{n}(C)$, построенной по кривой $L(C)$, где $C$ - некоторая связка аттрактора $\Omega$.

Для каждого номера $r \in\{1, \ldots, k\}$ построим замкнутую дугу $\lambda_{r}$ с граничными точками $\mu_{r}^{1}, \mu_{r}^{2}$, удовлетворяющую следуюшим условиям:

1) если для точки $p_{r}$ обе компоненты связности множества $W^{u}\left(p_{r}\right) \backslash p_{r}$ принадлежат $M_{i}$, то $z_{r} \in \operatorname{int} \lambda_{r}$ и точки $\mu_{r}^{1}, \mu_{r}^{2}$ принадлежат $L(C)$; в этом случае будем говорить, что построенная дуга имеет первыи тй

$2)$ если в точности одна из компонент связности множества $W^{u}\left(p_{r}\right) \backslash p_{r}$ принадлежит $M_{i}$, то одна из точек $\mu_{r}^{1}, \mu_{r}^{2}$ (для определенности будем считать, что это точка $\mu_{r}^{1}$ ) совпадает с точкой $z_{r}$, а другая принадлежит $L(C)$; в этом случае будем говорить, что построенная дуга имеет второй mun;

3) если дуга $\lambda_{r}$ имеет непустое пересечение с устойчивым многообразием точки, принадлежащей множеству $\Omega_{i}$, то это пересечение является трансверсальным.

Положим $\lambda=\bigcup_{r=1}^{k} \lambda_{r}$. По построению для каждой точки $z \in \lambda_{r}$ сушествует единственная точка $\widehat{z} \in W^{u}\left(p_{r}\right)$ такая, что точки $\widehat{z}, z$ принадлежат одному и тому же устойчивому многообразию некоторой точки из пересечения $\Omega_{i} \cap L(C)$ и дуга $(\widehat{z}, z)^{s}$ не пересекается с множеством $\bigcup_{r=1}^{k} W^{u}\left(p_{r}\right)$. Для любой точки $y \in[\widehat{z}, z]^{s}$ положим $\nu(y)=\widehat{z}$. В частности, имеем $\nu\left(\mu_{r}^{\delta_{r}}\right)=\widehat{\mu}_{r}^{\delta_{r}}\left(\delta_{r}=1,2\right), \nu\left(z_{r}\right)=p_{r}$.

Для каждого номера $r \in\{1, \ldots, k\}$ существует номер $\widetilde{r} \in\{1, \ldots, k\}$ такой, что кривая $f^{n_{0}}\left(\lambda_{\widetilde{r}}\right)$ пересекает кривую $L_{r}^{s}$. Положим $\widetilde{\lambda}_{r}=f^{n_{0}}\left(\lambda_{\widetilde{r}}\right)$ и $\widetilde{\lambda}=\bigcup_{r=1}^{k} \widetilde{\lambda}_{r}$.

Обозначим через $\eta_{r}^{1},\left(\eta_{r}^{2}\right)$ точку пересечения дуги $\widetilde{\lambda}_{r}$ с дугой $\left(\mu_{r}^{1}, \widehat{\mu}_{r}^{1}\right)^{s}\left(\left(\mu_{r}^{2}, \widehat{\mu}_{r}^{2}\right)^{s}\right)$ и через $\Pi_{r}$ замкнутую область, ограниченную дугами $\lambda_{r},\left[\mu_{r}^{1}, \eta_{r}^{1}\right] \subset\left[\mu_{r}^{1}, \widehat{\mu}_{r}^{1}\right]^{s}$, $\left[\eta_{r}^{1}, \eta_{r}^{2}\right] \subset \widetilde{\lambda}_{r},\left[\eta_{r}^{2}, \mu_{r}^{2}\right] \subset\left[\mu_{r}^{2}, \widehat{\mu}_{r}^{2}\right]^{s}$. Положим П $=\bigcup_{r=1}^{k} \Pi_{r}$. На рис. 8 показано построение областей П $1-\Pi_{5}$ для случая $k=5$. Здесь точки $p_{1}, p_{4}, p_{5}$ принадлежат связке степени $3 C$-плотной компоненты $\Omega_{i}$, точка $p_{2}=p_{3}$ принадлежит границе множества $M_{i}$, дуги $\lambda_{1}, \lambda_{4}, \lambda_{5}$ являются дугами первого типа, а дуги $\lambda_{2}, \lambda_{3}$ являются дугами второго типа.

Граничные точки $\mu_{r}^{\delta_{r}}, \mu_{r+1}^{\delta_{r+1}}$ кривых $\lambda_{r}, \lambda_{r+1}\left(\lambda_{k+1}=\lambda_{1}, \mu_{k+1}^{\delta_{k+1}}=\mu_{1}^{\delta_{1}}\right)$ назовем $s$-связаннылми, если

1) точки $\mu_{r}^{\delta_{r}}, \mu_{r+1}^{\delta_{r+1}}$ принадлежат одному и тому же устойчивому многообразию некоторой точки, принадлежащей $L(C) \cap \Omega_{i}$; 


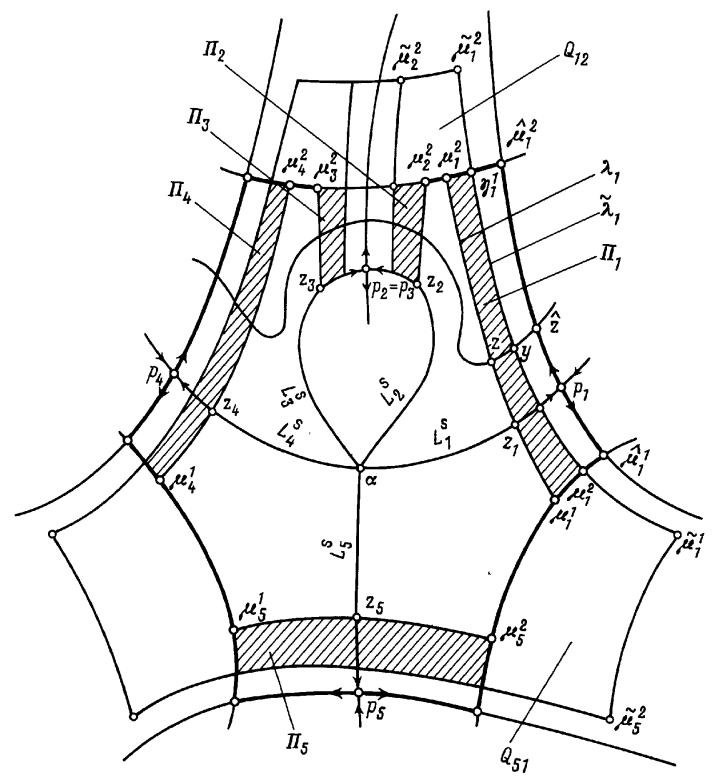

Рис. 8

2) $\left(\mu_{r}^{\delta_{r}}, \mu_{r+1}^{\delta_{r+1}}\right)^{s} \subset L(C)$;

3) $\left(\mu_{r}^{\delta_{r}}, \mu_{r+1}^{\delta_{r+1}}\right)^{s} \cap\left(\bigcup_{r=1}^{k} W^{u}\left(p_{r}\right)\right)=\varnothing$.

Дугу $\gamma_{r, r+1}=\left(\mu_{r}^{\delta_{r}}, \mu_{r+1}^{\delta_{r+1}}\right)^{s}$ назовем связывающей дугой для точек $\mu_{r}^{\delta_{r}}, \mu_{r+1}^{\delta_{r+1}}$.

Отметим, что по построению дуг $\lambda_{r}$ для каждой граничной точки $\mu_{r}^{\delta_{r}}$ найдется единственная точка либо $\mu_{r+1}^{\delta_{r+1}}$, либо $\mu_{r-1}^{\delta_{r-1}}\left(\mu_{k+1}^{\delta_{k+1}}=\mu_{1}^{\delta_{1}}, \mu_{0}^{\delta_{0}}=\mu_{k}^{\delta_{k}}\right)$ такая, что либо точки $\mu_{r}^{\delta_{r}}, \mu_{r+1}^{\delta_{r+1}}$, либо точки $\mu_{r-1}^{\delta_{r-1}}, \mu_{r}^{\delta_{r}}$ являются $s$-связанными, причем если дуга $\lambda_{r}$ первого типа, то $\delta_{r}$ может принимать два значения 1 и 2 , а если дуга $\lambda_{r}$ второго типа, то $\delta_{r}=2$.

Для точки $\mu_{r}^{\delta_{r}}$ обозначим через $\widetilde{\mu}_{r}^{\delta_{r}}$ граничную точку дуги $\widetilde{\lambda}_{r}$ такую, что дуга $\left[\widetilde{\mu}_{r}^{\delta_{r}}, \eta_{r}^{\delta_{r}}\right]$, принадлежашая $\widetilde{\lambda}_{r}$, не пересекается с дугой $\left(z_{r}, p_{r}\right)^{s}$.

Для связываюшей кривой $\gamma_{r, r+1}$ найдется связываюшая кривая $\widetilde{\gamma}_{r, r+1}$ такая, что кривая $\widetilde{\gamma}_{r, r+1}=f^{n_{0}}\left(\gamma_{r, r+1}\right)$ имеет точки $\widetilde{\mu}_{r}^{\delta_{r}}, \widetilde{\mu}_{r+1}^{\delta_{r+1}}$ своими граничными точками (рис. 8).

Обозначим через $Q_{r, r+1}$ замкнутую область, ограниченную дугами $\gamma_{r, r+1}$, $\left[\mu_{r+1}^{\delta_{r+1}}, \eta_{r+1}^{\delta_{r+1}}\right]^{s},\left[\eta_{r+1}^{\delta_{r+1}}, \widetilde{\mu}_{r+1}^{\delta_{r+1}}\right] \subset \widetilde{\lambda}_{r}, \widetilde{\gamma}_{r, r+1},\left[\widetilde{\mu}_{r}^{\delta_{r}}, \eta_{r}^{\delta_{r}}\right] \subset \widetilde{\lambda}_{r},\left[\eta_{r}^{\delta_{r}}, \mu_{r}^{\delta_{r}}\right]^{s}$.

Для каждой дуги $\lambda_{r}$ первого типа (и соответствующей дуги $\tilde{\lambda}_{r}$ ) обозначим через $\gamma_{r}\left(\widetilde{\gamma}_{r}\right)$ множество, состояшее из двух связывающих кривых, каждая из которых содержит по одной граничной точке $\mu_{r}^{1}, \mu_{r}^{2}$ дуги $\lambda_{r}\left(\widetilde{\mu}_{r}^{1}, \widetilde{\mu}_{r}^{2}\right.$ дуги $\left.\widetilde{\lambda}_{r}\right)$. Для каждой дуги $\lambda_{r}$ второго типа (и соответствующей дуги $\widetilde{\lambda}_{r}$ ) обозначим через $\gamma_{r}$ $\left(\widetilde{\gamma}_{r}\right)$ множество, состоящее из одной связываюшей кривой, содержашую граничную точку $\mu_{r}^{2}$ дуги $\lambda_{r}\left(\widetilde{\mu}_{r}^{2}\right.$ дуги $\left.\widetilde{\lambda}_{r}\right)$. Положим $\gamma=\bigcup_{r=1}^{k} \gamma_{r}$ и $\widetilde{\gamma}=\bigcup_{r=1}^{k} \widetilde{\gamma}_{r}$.

Для каждой дуги $\lambda_{r}$ первого типа с областью П $r$ имеют общую границу в точности две области $Q_{r, r+1}$ и $Q_{r-1, r}\left(Q_{k, k+1}=Q_{k, 1}, Q_{0,1}=Q_{k, 1}\right)$. Обозначим 
через $Q_{r}$ объединение этих областей. Для каждой дуги $\lambda_{r}$ второго типа с областью $\Pi_{r}$ граничит в точности одна область либо $Q_{r, r+1}$, либо $Q_{r-1, r}\left(Q_{0,1}=Q_{r, 1}\right)$. В этом случае обозначим через $Q_{r}$ эту область (на рис. 8 область $Q_{1}$, например, является объединением областей $Q_{12}$ и $Q_{51}$, а $Q_{2}$ совпадает с $\left.Q_{12}\right)$. Положим $Q=\bigcup_{r=1}^{k} Q_{r}$ и $K=\Pi \cup Q$.

Пусть теперь $\alpha^{\prime}$ источниковая периодическая точка диффеоморфизма $f^{\prime}$, изоморфная $\alpha$. Проведем для точки $\alpha^{\prime}$ те же построения, что и для $\alpha$, и снабдим обозначения изоморфных периодических точек, компонент связности устойчивых и неустойчивых многообразий седловых периодических точек, а также объектов, аналогичных объектам, построенным для точки $\alpha$, штрихами, сохранив прежние индексы. При этом, если $\mu_{r}^{1} \mu_{r}^{2}$ граничные точки кривой $\lambda_{r}$ и $\mu_{r}^{\prime 1}, \mu_{r}^{\prime 2}$ граничные точки кривой $\lambda_{r}^{\prime}$, то точки $\mu_{r}^{l}, \mu_{r}^{\prime l}$ принадлежат, соответственно, изоморфным компонентам связности множеств $W^{u}\left(p_{r}\right) \backslash p_{r}, W^{\prime u}\left(p_{r}^{\prime}\right) \backslash p_{r}^{\prime}$.

Выберем из каждой источниковой траектории диффеоморфизма $f_{i}$, принадлежащей множеству $U_{i}$, в точности по одной периодической точке. Обозначим через $\widetilde{U}_{i}$ множество всех таких точек и через $\widetilde{U}_{i}^{\prime}$ множество периодических точек диффеоморфизма $f_{i}^{\prime}$, состоящих из точек, изоморфных точкам из $\widetilde{U}_{i}$.

Условия изоморфизма различающих графов $G^{*}(f) G^{* *}\left(f^{\prime}\right)$ позволяют для каждой пары изоморфных точек $\alpha \in \widetilde{U}_{i}, \alpha^{\prime} \in \widetilde{U}_{i}^{\prime}$ построить гомеоморфизмы $h_{1}\left(\alpha, \alpha^{\prime}\right)$, $h_{2}\left(\alpha, \alpha^{\prime}\right), h_{3}\left(\alpha, \alpha^{\prime}\right), h_{4}\left(\alpha, \alpha^{\prime}\right), h_{5}\left(\alpha, \alpha^{\prime}\right), h_{6}\left(\alpha, \alpha^{\prime}\right)$, которые определяются следующим образом.

1) Гомеоморфизм $h_{1}\left(\alpha, \alpha^{\prime}\right)$ отображает множество П на множество П' и удовлетворяет следующему условию. Пусть $y$ произвольная точка из множества П и $y^{\prime}=h_{1}\left(\alpha, \alpha^{\prime}\right)(y)$. Тогда существуют номер $r$ и точки $z, z^{\prime}$, принадлежащие дугам $\lambda_{r}, \lambda_{r}^{\prime}$, соответственно, такие, что $y \in[\widehat{z}, z]^{s}$ (на рис. 8 указана точка $y$, для которой $r=1), y^{\prime} \in\left[\widehat{z}^{\prime}, z^{\prime}\right]^{s}$ и $\widehat{z}^{\prime}=H_{i}(\widehat{z})$.

2) Гомеоморфизм $h_{2}\left(\alpha, \alpha^{\prime}\right)$ отображает множество $\gamma$ на множество $\gamma^{\prime}$ и удовлетворяет условию $h_{2}\left(\alpha, \alpha^{\prime}\right)\left(\mu_{r}^{\delta_{r}}\right)=h_{1}\left(\alpha, \alpha^{\prime}\right)\left(\mu_{r}^{\delta_{r}}\right)$.

3) Гомеоморфизм $h_{3}\left(\alpha, \alpha^{\prime}\right)$ отображает множество $\widetilde{\lambda} \cup \widetilde{\gamma}$ на множество $\widetilde{\lambda}^{\prime} \cup \widetilde{\gamma}^{\prime}$ и определяется следуюшим образом. Для точки $x \in \widetilde{\lambda} \cup \widetilde{\gamma} h_{3}\left(\alpha, \alpha^{\prime}\right)(x)=$ $f_{i}^{\prime n_{0}} h_{1}\left(\alpha, \alpha^{\prime}\right) f^{-n_{0}}(x)$.

Из конструкции гомеоморфизма $h_{1}\left(\alpha, \alpha^{\prime}\right)$ следует, что

$$
\left.h_{3}\left(\alpha, \alpha^{\prime}\right)\right|_{\widetilde{\lambda} \cap \Pi}=\left.h_{1}\left(\alpha, \alpha^{\prime}\right)\right|_{\tilde{\lambda} \cap \Pi} \cdot
$$

4) Гомеоморфизм $h_{4}\left(\alpha, \alpha^{\prime}\right)$ отображает множество $K$ на множество $K^{\prime}$ и удовлетворяет условиям

$$
\begin{aligned}
\left.h_{4}\left(\alpha, \alpha^{\prime}\right)\right|_{\Pi} & =\left.h_{1}\left(\alpha, \alpha^{\prime}\right)\right|_{\Pi}, \\
\left.h_{4}\left(\alpha, \alpha^{\prime}\right)\right|_{\gamma} & =\left.h_{2}\left(\alpha, \alpha^{\prime}\right)\right|_{\gamma}, \\
\left.h_{4}\left(\alpha, \alpha^{\prime}\right)\right|_{\widetilde{\lambda} \cup \widetilde{\gamma}} & =\left.h_{3}\left(\alpha, \alpha^{\prime}\right)\right|_{\widetilde{\lambda} \cup \widetilde{\gamma}} .
\end{aligned}
$$

5) Гомеоморфизм $h_{5}\left(\alpha, \alpha^{\prime}\right)$ отображает множество $W^{u}(\alpha) \cap \bar{M}_{i}$ на множество $W^{\prime u}\left(\alpha^{\prime}\right) \cap \bar{M}_{i}^{\prime}$ и определяется следуюшим образом. Пусть $x \in W^{u}(\alpha) \cap \bar{M}_{i}$, тогда

$$
h_{5}\left(\alpha, \alpha^{\prime}\right)(x)=f^{\prime l n_{0}}\left(h_{4}\left(\alpha, \alpha^{\prime}\right)\left(f^{-l n_{0}}(x)\right)\right),
$$


где $l \in \mathbb{Z}$ такое, что $f^{-l n_{0}}(x) \in K$.

6) Гомеоморфизм $h_{6}\left(\alpha, \alpha^{\prime}\right)$ отображает множество

$$
\bigcup_{n=0}^{n_{0}-1} f^{n_{0}}\left(W^{u}(\alpha) \cap \bar{M}_{i}\right)
$$

на множество

$$
\bigcup_{n=0}^{n_{0}-1} f^{\prime n_{0}}\left(W^{\prime u}\left(\alpha^{\prime}\right) \cap \bar{M}_{i}^{\prime}\right)
$$

и определяется следуюшим образом. Если

$$
x \in \bigcup_{n=0}^{n_{0}-1} f^{n_{0}}\left(W^{u}(\alpha) \cap \bar{M}_{i}\right),
$$

то

$$
h_{6}\left(\alpha, \alpha^{\prime}\right)(x)=f^{\prime l}\left(h_{5}\left(\alpha, \alpha^{\prime}\right)\left(f^{-l}(x)\right)\right),
$$

где $l \in\left\{0, \ldots, n_{0}-1\right\}$ такое, что $f^{-l}(x) \in W^{u}(\alpha) \cap \bar{M}_{i}$.

Непосредственно проверяется, что гомеоморфизм $h_{6}\left(\alpha, \alpha^{\prime}\right)$ удовлетворяет условию

$$
\left.f_{i}^{\prime}\right|_{\cup_{n=0}^{n_{0}-1} f^{\prime n_{0}\left(W^{\prime u}\left(\alpha^{\prime}\right)\right)}}=\left.h_{6}\left(\alpha, \alpha^{\prime}\right) f_{i} h_{6}^{-1}\left(\alpha, \alpha^{\prime}\right)\right|_{\bigcup_{n=0}^{n_{0}-1} f^{\prime n_{0}}\left(W^{\prime u}\left(\alpha^{\prime}\right)\right)} .
$$

Пусть теперь $x \in \bar{M}_{i}$, тогда либо $x \in S_{i} \cup U_{i} \cup W_{* i}^{u}$, либо найдется точка $\alpha \in \widetilde{U}_{i}$ периода $n_{0}$ такая, что

$$
x \in \bigcup_{n=0}^{n_{0}-1} f^{n_{0}}\left(W^{u}(\alpha) \cap \bar{M}_{i}\right) .
$$

Положим

$$
H_{i}(x)= \begin{cases}h_{6}\left(\alpha, \alpha^{\prime}\right)(x), & x \in \bigcup_{n=0}^{n_{0}-1} f^{n_{0}}\left(W^{u}(\alpha) \cap \bar{M}_{i}\right), \\ h_{1}(x), & x \in S_{i} \cup U_{i} \cup W_{* i}^{u} .\end{cases}
$$

Построенное отображение удовлетворяет условию $\left.f_{i}\right|_{\bar{M}_{i}^{\prime}}=\left.H_{i} f H_{i}^{-1}\right|_{\bar{M}_{i}^{\prime}}$. Покажем, что отображение $H_{i}$ является гомеоморфизмом. Достаточно показать, что отображение $H_{i}$ непрерьвно в любой точке $x$, принадлежащей неустойчивому многообразию любой точки из $W_{i *}^{u}$.

Из вышеописанной конструкции гомеоморфизма $H_{i}$ следует, что сушествуют целое неотрицательное число $N$, источниковая, периодическая точка $\alpha \in \widetilde{U}_{i}$, номер $r$, точки $p_{r} \in S_{i}, z \in \operatorname{int} \lambda_{r}$ такие, что $f_{i}^{N}(x) \in W^{u}\left(p_{r}\right), \nu(z)=f_{i}^{N}(x)$. Пусть $\left\{x_{k}\right\}$ последовательность точек из $M_{i}$, сходяшаяся к точке $x$. Положим $y=f_{i}^{N}(x)$, $y_{k}=f_{i}^{N}\left(x_{k}\right)$. Не уменшшая общности, можно предполагать, что для каждой точки $y_{k}$ найдется точка $z_{k} \in \lambda_{r}$ такая, что $y_{k} \in\left(\nu\left(z_{k}\right), z_{k}\right)^{s}$.

Положим $y^{\prime}=H_{i}(y), y_{k}^{\prime}=H_{i}\left(y_{k}\right)$. Из непрерывной зависимости устойчивых многообразий точек из множества $\Omega_{i}$ на компактных множествах следует, что последовательность точек $\left\{z_{k}\right\}$ сходится к точке $z$. Положим $z^{\prime}=H_{i}(z), z_{k}^{\prime}=$ 
$H_{i}\left(z_{k}\right)$. Из конструкции гомеоморфизма $h_{6}\left(\alpha, \alpha^{\prime}\right)$ следует, что $y_{k}^{\prime} \in\left(\nu\left(z_{k}^{\prime}\right), z_{k}^{\prime}\right)^{s}$. Так как отображение $h_{1}$ является гомеоморфизмом, то последовательность $\left\{z_{k}^{\prime}\right\}$ сходится к точке $z^{\prime}$, и следовательно, из близости устойчивых многообразий точек из множества $\Omega_{i}^{\prime}$ на компактных множествах последовательность $\left\{\nu\left(z_{k}^{\prime}\right)\right\}$ сходится к точке $\nu\left(z^{\prime}\right)$. Но тогда последовательность $\left\{y_{k}^{\prime}\right\}$ сходится к точке $y$.

Так как

$$
H_{i}\left(x_{k}\right)=H_{i}\left(f_{i}^{-N}\left(y_{k}\right)\right)=f_{i}^{\prime-N}\left(H_{i}\left(y_{k}\right)\right),
$$

то $\left.H_{i}\left(x_{k}\right)\right)$ сходится к $f^{\prime-N}\left(H_{i}(y)\right)=H_{i}\left(f_{i}^{-N}(y)\right)=H_{i}(x)$. Таким образом, отображение $H_{i}$ является непрерьвньп всюду на $\bar{M}_{i}$.

Зададим теперь отображение $h_{\Omega}$ множества $\bar{M}_{\Omega}$ на множество $\bar{M}_{\Omega^{\prime}}^{\prime}$ следующим образом. Для точки $x \in \bar{M}_{\Omega}$ положим $h_{\Omega}(x)=f^{\prime l}\left(h_{i}\left(f^{-l}(x)\right)\right)$, где $l \in\{0, \ldots, m-1\}$ такое, что $f^{-l}(x) \in \bar{M}_{i}$. Построенный таким образом гомеоморфизм $h_{\Omega}$ удовлетворяет условиям $\left.h_{\Omega}\right|_{\Omega}=\left.g\right|_{\Omega}$ и $\left.f\right|_{M_{\Omega^{\prime}}^{\prime}} ^{\prime}=\left.h_{\Omega} f h_{\Omega}^{-1}\right|_{\bar{M}_{\Omega^{\prime}}^{\prime}} ^{\prime}$. Лемма 11 полностью доказана.

Лемма 12. Пусть различающие графь $G^{*}(f), G^{*}(f)$ диффеоморфизмов $f, f^{\prime} \in S(M)$ изоморфны в силу изоморфизма $\eta$ такого, ито $P^{\prime}\left(f^{\prime}\right)=\eta P(f) \eta^{-1}$. Тогда существует гомеоморфизм $H$ многообразия $M$ на себя такой, что $f^{\prime}=H f H^{-1}$.

ДокАЗАТЕЛЬСтво. Зададим гомеоморфизм $h_{\Lambda}$ множества $\bar{M}_{\Lambda}$ на множество $\bar{M}_{\Lambda^{\prime}}^{\prime}$ следующим образом. Пусть $x$ произвольная точка, принадлежащая множеству $\Lambda$. Обозначим через $\Omega(x)$ одномерное базисное множество, содержашее точку $x$, и положим $h_{\Lambda}(x)=h_{\Omega(x)}(x)$.

Пусть $\Sigma=M \backslash M_{\Lambda}, \Sigma^{\prime}=M \backslash M_{\Lambda}^{\prime}$. Применим к ограничениям диффеоморфизмов $f$ и $f^{\prime}$ на множества $\Sigma$ и $\Sigma^{\prime}$ конструкцию построения сопрягающего гомеоморфизма $h_{\Sigma}$, описанную в работе [19]. Отметим, что эта конструкция позволяет построить $h_{\Sigma}$ таким образом, что $\left.h_{\Sigma}\right|_{\partial M_{\Lambda}}=\left.h_{\Lambda}\right|_{\partial M_{\Lambda}}$.

Зададим гомеоморфизм $H$ многообразия $M$ на себя формулой

$$
H(x)= \begin{cases}h_{\Lambda}(x), & x \in M_{\Lambda} \\ h_{\Sigma}(x), & x \in M_{\Sigma}\end{cases}
$$

Тогда $H$ является искомьм гомеоморфизмом. Лемма 12 доказана.

\section{Список литературы}

1. Palis J. On Morse-Smale dynamical systems // Topology. 1969. V. 8. №4. P. 385-404.

2. Пали Дж., Смейл C. Теоремы структурной устойчивости // Математика. 1969. Т. 13. № 2. C. $145-155$.

3. Robbin J. W. A structural stability theorem // Ann. of Math. 1971. V. 94. № 3. P. 447-493.

4. Sannami $A$. The stability theorems for discrete dynamical systems on two-dimensional manifolds // Proc. Japan Acad. Ser. A. Math. Sci. 1981. V. 57. P. 403-407; // Nagoya Math. J. 1983. V. 90. P. 1-55.

5. Liao S. T. On the stability conjecture // Chinese Ann. of Math. 1980. V. 1. № 1. P. 9-30.

6. Mane R. An ergodic closing lemma // Ann. of Math. 1982. V. 116. №6. P. 503-540. 
7. Smale S. Morse inequalities for a dynamical systems // Bull. Amer. Math. Soc. 1960. V. 66. P. $43-49$.

8. Гринес В.З. О топологической сопряженности диффеоморфизмов двумерного многообразия на одномерных базисныт множествах // УМН. 1974. Т. 29. №6. С. 163-164.

9. Гринес В.З. О топологической сопряженности диффеоморфизмов двумерного многообразия на одномерных ориентируемых базисных множествах. I // Труды MМО. 1975. T. 32 . C. $35-61$.

10. Гринес В.З. О топологической сопряженности диффеоморфизмов двумерного многообразия на одномерных ориентируемых базисных множествах. II // Труды MМО. 1977. T. 34. C. $243-252$.

11. Плькин Р. В. О геометрии гиперболических аттракторов гладких каскадов // УМН. 1984. T. 39. №6. C. 75-113.

12. Гринес В.З., Калай X. X. О топологической эквивалентности диффеоморфизмов с нетривиальными базисньми множествами на двумерных многообразиях // Методы качественной теории дифференц. уравнений. / ред. Е.А. Леонтович-Андроновой. Горький: Горьк. гос. ун-т, 1988. С. 40-49.

13. Гринес В. З.,Калай $X$. X. Топологическая классификация базисных множеств без пар сопряженных точек $A$-диффеоморфизмов на поверхностях // Деп. ВИНИТИ. 1988. № 1137-В. Горький.

14. Арансон С.X., Гринес В. З. Топологическая классификация каскадов на замкнутых двумерных многообразиях // УМН. 1990. Т. 45. № 4. С. 3-32.

15. Арансон C.X., Гринес В. З. Каскады на поверхностях. Динамические системы с гиперболическим поведением // Итоги науки и техники. Совр. проблемы матем. Фундаментальные направления. Т. 66. М.: ВИНИТИ, 1991. С. 148-187.

16. Жиров А. Ю. Гиперболические аттракторы диффеоморфизмов ориентируемых поверхностей. Часть 1. Кодирование, классификация и накрытия // Матем. сб. 1994. Т. 185. №6. С. $3-50$.

17. Жиров А. Ю. Гиперболические аттракторы диффеоморфизмов ориентируемых поверхностей. Часть 2. Перечисление и применение к псевдоаносовским диффеоморфизмам // Матем. сб. 1994. Т. 185. №9. С. 29-80.

18. Жиров А. Ю. Гиперболические аттракторы диффеоморфизмов ориентируемых поверхностей. Часть 3. Алгоритм классификации // Матем. сб. 1995. Т. 186. № 9. С. 59-82.

19. Гринес В.З. Топологическая классификация диффеоморфизмов Морса-Смейла с конечным множеством гетероклинических траекторий на поверхностях // Матем. заметки. 1993. T. 54. № 3. С. 3-17.

20. Смейл С. Дифференцируемые динамические системы // УМН. 1970. Т. 25. № 1. C. $113-185$.

21. Безденежных A. Н., Гринес В. З. Динамические свойства и топологическая классификация градиентноподобных диффеоморфизмов на двумерных многообразиях // Методы качественной теории дифференц. уравнений / ред. Е. А. Лентович-Андроновой. Горький: Горьк. гос. ун-т, 1985. С. 22-38.

22. Безденежсных A.H., Гринес B.З. Диффеоморфизмы с ориентируемыми гетероклиническими множествами на двумерных многообразиях // Методы качественной теории дифференц. уравнений / ред. Е.А. Лентович-Андроновой. Горький: Горьк. гос. ун-т, 1985. С. $139-152$.

23. Безденежных А. Н., Гринес В. З. Динамические свойства и топологическая классификация градиентноподобных диффеоморфизмов на двумерных многообразиях. Часть 2 // Методы качественной теории диффференц. уравнений / ред. Е. А. Лентович-Андроновой. Горький: Горьк. гос. ун-т, 1987. С. 24-32.

24. Безденежных A.H., Гринес B.З. Реализация градиентноподобных диффеоморфизмов двумерных многообразий // Дифференциальные и интегральные уравнения / ред. Н. Ф. Отрокова. Горький: Горьк. гос. ун-т, 1985. С. 33-37. 
25. Боревич E. А. Условия топологической эквивалентности двумерных диффеоморфизмов Морса-Смейла // Изв. вузов. Сер. матем. 1980. № 11. С. 12-17.

26. Боревич Е. А. Условия топологической эквивалентности двумерных дифффеоморфизмов Морса-Смейла // Дифференц. уравнения. 1981. Т. 17. № 8. С. 1481-1482.

27. Боревич E. A. Топологическая эквивалентность двумерных диффеоморфизмов Морса-Смейла // Изв. вузов. Сер. матем. 1984. № 4. С. 3-6.

28. Боревич E. A. Двумерные диффеоморфизмы Морса-Смейла, имеющие ориентированные гетероклинические связи // Изв. вузов. Сер. матем. 1989. № 9. С. 77-79.

29. Аносов Д. В. Геодезические потоки на замкнутых римановых многообразиях отрицательной кривизны // Труды МИАН. 1967. Т. 90.

30. Аносов Д. В. Об одном классе инвариантных множеств гладких динамических систем // Труды пятой международной конференции по нелинейным колебаниям. Т. 2. Качественные методы. Ин-т математики АН УССР. Киев: Наукова Думка, 1970. С. 39-45.

31. Bowen R. Periodic points and measures for axiom A diffeomorfisms // Trans. Amer. Math. Soc. 1971. V. 154. P. 337-397.

32. Плькин Р. В. О топологии базисных множеств диффеоморфизмов С. Смейла // Матем. сб. 1971. Т. 84. № 2. С. 301-312.

33. Плькин Р. В. Источники и стоки $A$-диффеоморфизмов поверхностей // Матем. сб. 1974. T. 94. № 2. C. 243-264.

34. Robinson R. S., Williams R.F. Finite stability is not generic // Proc. of a symp. held on the Univ. of Brasil. 1971. New York: Acad. Press, 1973. P. 451-462.

35. Nielsen J. Surface trnsformation classes of algebraically finite type // Det. Kgl. Dansk Videnskaternes Selskab. Math.-Phys. Meddelerser. 1944. V. 21. P. 1-89.

Нижегородская государственная

Поступила в редакцию

сельскохозяйственная академия

16.10.1996 